\title{
Sulla differenziabilità totale delle funzioni di più variabili reali.
}

\author{
Memoria di Franchsco Severi (a Roma).
}

Sunto. - Sul fondamento delle nozioni (altrove introdotte dall'A.) di tangenti e corde improprie d'un insieme di punti in un punto d'accumulazione, si deterninann condizioni necessarie e sufficienti per la differenziabilitì o per l' iperafferenziabilitò di una funzione di pit variabili in un punto d'accumulazione d'un insiene o all' interno di un dominio in cui essa sia defwita. La consueta considerazione delle derivate paraiali non esaurisce nè può esaurire la questione. Percì̀ l'A. deve anzititto defunire l'operazione di derivazione per una funzione di più variabili in modo adegnato alla natura della funzione, la quale esige che non ci si liniti a studiar la destivazione rispetto alle variabili separatamente considerate.

L'argomento è di quelli studiati e ristudiati fin dagl'inizi della revisione critica dell'Analisi $\left({ }^{1}\right)$. Mi sembra tuttavia che possan ancora trovar posto le considerazioni che seguono, sia perchè esse conducono, in modo elementare, a condizioni necessarie e sufficienti per la differenziabilità o per l'iperdifferenziabilità in un punto $P_{0}$ (mentre le condizioni note riguardan la differenziabilita in quasi tutti i punti d' un insieme misurabile); sia perchè l'insieme in cui la funzione è data è vincolato dalla sola condizione che $P_{0}$ ne sia un punto d' accumulazione (sicchè in $P_{0}$ le derivate parziali non posson generalmente considerarsi); sia infine perchè dal presente lavoro risulta una condizione necessaria e sufficiente per la differenziabilità o per l'iperdifferenziabilità in tutti i punti di un dominio.

La differenziabilità in $P_{0}$ di una funzione $f(P)$ di un punto $P$ variabile in un insieme piano serrato $I$ (mi limito alle funzioni di due variabili, faeile presentandosi l'estensione ad $n>2$ variabili), è intesa nel senso generale di SToLz, cioè nel senso che esista un' espressione $\alpha \Delta x+\beta \Delta y$, con $\alpha, \beta$ costanti, tale che:

$$
\lim \frac{1}{P_{0} P}\left[f(P)-f\left(P_{0}\right)-\alpha \Delta x-\beta \Delta y\right]=0
$$

(1) Indicazioni bibliografiche aggiornate si leggono nel t. Ir delle monografie matematiche di Varsavia, Théorie te l'integrale, doruto a S. Saks (Varsavia, 1933, pag. 222 e segg.). Pei rapporti di questa mia Memoria con una recentissima di G. Guaresch red. i nn. 1; 5. Oss.; 16 . 
per $P$ comunque tendente a $P_{0}$ in $I ; \Delta x, \Delta y$ denotando le componenti del vettore $P_{\|} P$.

Intendo invece per iperdifferenziale $\alpha \Delta x+\beta \Delta y$ un espressione tale che:

$$
\lim \frac{1}{Q P}[f(P)-f(Q)-\alpha \Delta x-\beta \Delta y]=0
$$

per $P, Q$ distinti, tendenti comunque a $P_{0}$ in $I ; \Delta x, \Delta y$ denotando le componenti del vettore $Q P$.

Allo scopo di ottenere le desiderate condizioni, introduco i concetti di derivate, ultraderivate, iperderivate direzionali di $f(P)$ in $P_{0} . V i$ son funzioni elementari che hanno derivate direzionali e non ultraderivate nè iperderivate; funzioni che hanno derivate e ultraderivate e non iperderivate. Quando esistono le ultraderivate, esistono le derivate ed hanno valori uguali nelle stesse direzioni; quando esistono le iperderivate, esistono le ultraderivate e le derivate ed hanno valori uguali nelle stesse direzioni.

Le condizioni cui ho alluso divengon particolarmente significative allorchè si tratta della differenziabilità e dell' iperdifferenziabilità nei punti di un dominio piano $I$. Poichè si dimostra che le iperderivate, quando esistono in un dominio piano, son necessariamente continue, ne deriva che la condizione necessaria e sufficiente (la sufficienza è ovvia!) per l'jperdifferenziabilità all'interno di $I$ è che esistano in ogni punto interno ad $I$ le derivate parziali e sieno continue. Vale naturalmente la proprietà analoga per le funzioni di una variabile. Dal punto di vista geometrico questi fatti s'esprimon così :

Una linea piana di Jordan con tutti i suoi punti semplici ha la tangente continuta.

Conformemente alla definizione generale, che ho altrove dato pei punti semplici di una varietà topologica (ved. le citazioni in seguito) un-punto $P$ della linea è semplice se esiste in esso una sola tangente e se una retta qualsiasi, con direzione diversa da quella della tangente, vicina a $P$, incontra la curva attorno a $P$ in un punto al più (esattamente ì un punto, se $P \dot{e}$ interno alla linea, al più in uno, se $P$ è un estremo).

Una superficie di Jordan con tutti $i$ suoi punti semplici ha il piano tangente continuo.

Un punto $P$ della superficie è semplice quando le tangenti giaceiono in un piano e una retta vicina a $P$, non parallela a questo piano, incontra la superficie attorno a $P$ in un punto al più ; ecc..

Per ciò che concerne la semplice differenziabilità in un punto $P_{\mathrm{n}}$ interno ad un dominio piano $I$, dove sia data $f(P)$, le considerazioni dei nn. 21, 22, 23 mostrano che, a voler tenere nel debito conto la proprietà di $f(P)$ come fun- 
zione della coppia $(x, y)$ e non delle variabili prese separatamente, oceorre svincolarsi dall'ordinaria nozione di derivate parziali, per le quali bisogna prescrivere modi troppo particolari di variabilita di $P$ verso $P_{0}$.

Sembra che convenga meglio alla natura dell'ente variabile indipendente (che è una coppia di variabili simultanee), di assumer come derivatè parziali i limiti dei rapporti incrementali

$$
\frac{f(x, y)-f\left(x_{0}, y\right)}{x-x_{0}}, \frac{f(x, y)-f\left(x, y_{0}\right)}{y-y_{0}}
$$

(in cui la $y$ o rispettivamente la $x$ è la stessa nei due termini del numeratore), quando $P(x, y) \rightarrow P_{0}\left(x_{t}, y_{0}\right)$ in modo che $\lim P_{0} P$ sin una semiretta $\lambda$, per cui non sia rispettivamente cosiante $x$ od $y$; sempre che, naturalmente, questi limiti esistano finiti e indipendenti da $\lambda$.

S'ottengon così le derivate parziali generalizzate. Ebbene la loro esistenza in $P_{n}$ è condizione necessaria e sufficiente per la differenziabilità. Esse dunque estendono, nel modo più appropriato, il concetto di derivata delle funzioni di una variabile, la eui esistenza equivale appunto alla differenzinbilità.

La continuità superficiale di $f$ è conseguenza del solo fatto che gl'inerementi parziali generalizzati $f(x, y)-f\left(x_{0}, y\right), f(x, y)-f\left(x, y_{0}\right)$ tendano a zero, per $P \rightarrow P_{0}$ nelle condizioni precedenti: il che dà una riprova dell'opportunità della loro considerazione.

Una condizione sufficiente, singolarmente espressiva, per la differenziabilita di $f(P)$ in tutti i punti interni ad un dominio piano $I$, si ottiene supponendo che i limiti $\alpha\left(x_{n}, y_{n}\right), \beta\left(x_{v}, y_{0}\right)$ dei predetti rapporti esistano finiti, per $P$ tendente a $P_{0}$ sopra una retta qualunque uscente da $P_{0}$ (nella quale non sia $x=$ cost. o rispettivamente $y=$ cost.), e siano indipendenti dalla retta mede. sima, purehè si aggiunga l'ipotesi che le funzioni $\alpha\left(x_{0}, y_{0}\right), \beta\left(x_{0}, y_{0}\right)$ si conser. vino limitate in ogni dominio tutto interno ad $I$.

Questo risultato ha una certa analogia col bel teorema di RADEMAOHER che una $f(P)$, le cui derivate parziali si conservin limitate in un dominio piano, è ivi quasi dappertutto differenziabile. Pero per esser sicuri che la funzione sia ovunque differenziabile bisogna come vedesi passare dai rapporti incrementali parziali ordinari ai rapporti incrementali generalizzati $\left({ }^{2}\right)$.

(1) Aleme delle considerazioni stolto nella presente Memoria son legate (a preseinder dalla forma) ni conceti sriluppati da $G$. Bovugand in un suo interessante libro recente (Introluction à la géonétrie infinitésimale directe, Paris, Vuibert, 1932), come indichero con maggior precisione in seguito. Le nozioni di semitangenti e di corde improprie di un insieme di punti in un punto di accumnlazione, che stanno a fondamento del mio lavoro, corrispon. dono a quelle di contingente e di paratingente di BOUligand. All' egregio guometra è evi. 


\section{Tangenti e corde improprie in un punto d'accumulazione d'un insieme.}

1. Richiamo anzitutto le nozioni di tangenti, semitangenti, corde improprie di un insieme qualunque $I$ di punti di un $S_{n}$ (reale), in un punto d'accumulazione $O$, da me introdotte in precedenti lavori ( $(3)$.

Una semiretta $a$ di origine $O$ dicesi semitangente in $O$ ad $I$ quando essa contiene infiniti punti di $I$, accumulati attorno ad $O$, oppure quando, scelto $\varepsilon>0$ e determinato l'insieme $K_{\varepsilon}$ delle semirette proiettanti da $O$ i punti di $I$ diversi da $O$, situati nell'intorno di raggio $\varepsilon$, la $\alpha$ è di accumulazione per $K_{\varepsilon}$ qualunque sia $\varepsilon$. Le due alternative posson anche presentarsi insieme. Una tangente è una retta contenente una semitangente.

Una retta $b$ per $O$ dicesi una corda impropria di $I$ quando essa contiene infiniti punti di $I$ attorno ad $O$, oppure quando, scelto $\varepsilon>0$, e considerato l'insieme $H_{\varepsilon}$ delle rette che congiungon coppie di punti di $I$, diversi tra loro, situati nell'intorno di raggio $\varepsilon, b$ di accumulazione per $H_{\S}$, qualunque sia $\varepsilon$. Le due alternative posson presentarsi insieme.

Ho dimostrato che in $O$ vi son sempre semitangenti e corde improprie. Una tangente è una corda impropria; ma non necessariamente viceversa.

dentemente sfuggito che le sue ricerche in proposito sono state inizjate un po' più tardi delle mie: il che ho gia aruto occasione di porre in rilievo nella Nota di Cracovia, più sotto eitata. (Anche la nozione di distanea di due insiemi di punti, esposta al $\mathbf{n}$. 34 del libro di Bovrigand, trovarasi gì in varii lavori miei : "Rendiconti dei Lincei », 1927, p. 476; 1929, p. 918; Conferenze di geometria algebrica, Roma, 1927-30, p. 122; Conferenze sulla topologia tenute a Buenos Aires, Imprenta de la Universidad, 1930, pag. 28). Ma non gli muovo im. provero per questo, perché neppur io riesco a seguire con cnra minuziosa la bibliografia e leggo più volentieri una memoria o un libro dopo aver pensato per eonto mio all' argomento.

(3) Ved. la mia più recente Nota in proposito: Su alcune questioni di topologia infinitesimale ("Annales de la Societé polonaise de Math.», t. IX, 1930). Iri si troveranno anche i riferimenti alle ricerche di Bovmigand. Il Guaresciu nell' interessante laroro: Un concetto di derivazione delle funzioni di pià variabiti reali, ecc. ("Memorie della R. Accademia d'Italia ${ }^{2}$ 1984-XII, p. 173) ha dedotto dai concetti che sto per richiamare un'espressiva condizione geometrica per la differenziabilita totale di una funzione di pin variabili in nu punto. Il teorema del GUAmeson è ritrovato come corollario alla fine del n. 5 di questa Memoxia. Il teorema medesimo ha consentito all'A. di estendere la considerazione delle derivate parziali, in guisa da ottenere enti più generali (ch' egli chiama derivate perfette. ved. n. 16 della presente Memoria), i quali esistono e posson introdursi nel ealeolo, anche quando non vi sono le dexivate paralia ordinarie. Ver. altresi, a proposito del significato goometrico dell' esistenza del differenziale totale, il n. 72 del libro di Bouligand. 
Inoltre, se l'insieme $I$ è serrato $\left.\left.\right|^{4}\right)$ : ogni semitangente puo definirsi come il limite per $n \rightarrow \infty$ della semiretta $O P_{n}$, ove $P_{n}$ percorra una conveniente successione di punti di $I$ convergente ad $O$; ogni corda impropria $\grave{e}$ il limite per $n \rightarrow \infty$ della retta $P_{n} Q_{n}$, ove i punti distinti $P_{n}, Q_{n}$ percorran due con venienti successioni convergenti ad $O$ tuna delle quali può ridursi al punto fisso $O$, se la corda è tangente).

2. Converrà qui distinguere le corde dalle semicorde improprie, cioè dalle semirette in eui le corde son divise da $O$. Dimostriamo che:

Data una semicorda impropria, esiston sempre nell insieme I due succes. sioni $P_{n}, Q_{n}$ convergenti ad $O$, tali che, per ogni $n, P_{n}, Q_{n}$ son distinti; cia* scuna delle dne successioni ha in $O$ una sola semitangente e la semiretla $P_{n} Q_{n}$ ha per limite la data semicorda.

Sia invero $\lambda$ la data semicorda ed $l$ la corda impropria che la contiene. Limitiamo le dne successioni a quei punti, d'un intorno abbastanza ristretto di $O$, tali che le rette $A_{n} B_{n}$ fove le successioni $A_{n}, B_{n}$ convergono ad $O$ in guisa che $\lim A_{n} B_{\imath}=l$ ), formino cen $l$ un angolo (acuto) minore d'un angolo prefissato $\delta$. Nessuna delle rette $A_{n} B_{n}$ sarà allora parallela ad un dato iperpiano $\Omega$ passante per $O$, ma non per $l$; epperò si può su ognuna di esse determinare un verso che abbia per limite il verso di $\lambda$ quando $A_{n} B_{n}$ tende ad $l$. Coi punti $A_{n}, B_{n}$ posson in conseguenza formarsi due nuove successioni $A_{n}{ }^{\prime}, B_{n}{ }^{\prime}$ ponendo nella prima quello dei due punti $A_{n}, B_{n}$ che precede l'altro nel verso fissato. In tal modo risulta $\lambda=\lim A_{n}{ }^{\prime} B_{n}{ }^{\prime}$.

Se la successione $A_{n}{ }^{\prime}$ ha in $O$ più di una semitangente, è possibile estrarre da essa una successione parziale $A_{n}$ ", che abbia in $O$ una sola semitangente $\lambda_{1}$. Chiamiamo $B_{n}{ }^{\prime \prime}$ l'omologo di $A_{\imath}{ }^{\prime \prime}$ nella successione $B_{\imath}{ }^{\prime}$. Se la successione $B_{n}{ }^{\prime \prime}$ ha in $O$ più di una semitangente, si potrà da essa estrarre una successione parziale $Q_{n}$, che abbia in $O$ una sola semitangente $\lambda_{2}$. Diciamo $P_{n}$ il punto omologo di $Q_{n}$ nella successione $A_{n}{ }^{\prime \prime}$. Allora le successioni $P_{n}$, $Q_{n}$ soddisfanno al teorema enunciato.

3. Le semitangenti $\lambda_{1}, \lambda_{2}$ alle successioni $P_{n}, Q_{n}$ del n. prec. posson benissimo essere allineate (cioè coincidenti od opposte). Perchè si possan costruire le successioni stesse in modo che $\lambda_{1}, \lambda_{2}$ non risultino allineate, basta che due

(4) Ciò̀ contiene tutti i propri punti d'accumulazione. L'ipotesi che $I$ sia serrato diventa superflua, ora e nel seguito, se si ammette che abbia senso l'operazione di formare una ben definita successione togliendo un punto da ciascun insieme di una successione d"in. siemi di punti. 
delle tangenti alle successioni iniziali $A_{n}, B_{n}$ sieno distinte (il che accude sempre che una delle successioni abbia più di una tangente in 0 ).

Puó darsi però che, comunque si costruiscano le successioni $P_{n}, Q_{n}$ soddisfacenti al teorema del n. 2, esse abbian di necessità in $O$ la medesima tau. gente. Questo avviene di certo quando $I$ ha in $O$ una sola tangente.

A noi importa invece di considerare il caso in eni per ogni semicorda $\lambda$ si posson determinare due successioni $P_{n}, Q_{n}$ che abbiano in $O$ tangenti distinte. Definiremo percio quelli che chiameremo punti di accumulazione regolare (rispetto al concetto di semicorda impropria).

Il punto $O$ si chiamerà di accnmulazione regolare quando scelta comunque una semitangente $x$ e una semicorda impropria $\lambda$ di $I$ in $O$, può determinarsi (almeno) una semitangente $y$ non allineata con $x$, tale che $\lambda$ sia ottenibile come limite della semiretta $P Q$ per $P$, $Q$ tendenti ad $O$ sotto le condizioni $\lim O P=x, \lim O Q=y$.

Ogni punto $O$ interno ad un dominio piano $I$ è di accumulazione regolare; ed è altresì di accumulazione regolare ogni punto contorno del dominio piano racchiuso da una linen di JoRDAN a punti trtti semplici (ofr. col. n. 18). Se invece la linea ha p. es. una cuspide, ivi cade un'accumulazione irregolare del dominio.

\section{Differenziali e derivate direzionali.}

4. Mi riferisco alle funzioni di due variabili reali $x, y$, giacchè facili si presentan le estensioni alle funzioni di $n$ variabili.

Per snellire l'esposizione alteriore occorre il seguente elementarissimo lemma : «Se

$$
z-z_{0}=\alpha\left(x-x_{0}\right)+\beta\left(y-y_{0}\right)
$$

ossia $z=\varphi(M)$, ove $M$ è un punto mobile sul piano $x y$ è l'equazione di un piano non parallelo a $z(x, y, \approx$ assi cartesiani anche obliqui) e $P, Q$ son due punti del piano $x y$ variabili in un insieme serrato $I$, che abbia un'accumn. lazione in $P_{0}$, risulta

$$
\lim \frac{\varphi(P)-\varphi(Q)}{Q P}=\alpha \frac{\sin \lambda y}{\sin x y}+\beta \frac{\operatorname{si} x \lambda}{\sin x y},
$$

ogni volta che $P, Q$ tendano a $P_{\xi}$ in modo che la semiretta $Q P$ tenda ad una semicorda impropria $\lambda$ di $I$ in $P_{0} »$.

S' intende che $Q P$ denota il valore assoluto della distanza dei due punti, sicchè la retta $Q P$ va orientata positivamente da $Q$ verso $P$. 
Si ha infatti dalla (1):

$$
\frac{\varphi(P)-\varphi(Q)}{Q P}=\alpha \frac{\Delta x}{Q P}+\beta \frac{\Delta y}{Q P},
$$

ove $\lrcorner x, \Delta y$ son le componenti del vettore $Q P$; e siccome, denotata con $r$ la semiretta $Q P$ sussistono (in valore e segno rispetto ai versi delle rette considerate e al verso positivo delle rotazioni su $x y$ ), le:

$$
\frac{\Delta x}{Q P}=\frac{\sin r y}{\sin x y}, \quad \frac{\Delta y}{Q P}=\frac{\sin x r}{\sin x y} .
$$

passando al limite per $P, Q \rightarrow P_{0}$ si ottiene la (2).

In particolare, la conclusione vale quando $Q$ è fisso e coincidente con $P_{0}$, sicchè $\lambda$ è semitangente ad $I$ in $P_{0}$.

5. La differensiabilitò (si sottintende totale) di una funzione reale $f(x, y)$ o $f(P)$, si può intendere da due punti di vista: uno ristvetto ed uno lato.

La $f(P)$ sia data, finita, in un insieme serrato $I$ avente un' accumulazione in $P_{0}$ e sia continua in $P_{0}$. Dal punto di vista ristretto si può considerare come differenziale tolale di $f(P)$ in $P_{0}$ un' espressione $\alpha \Delta x+\beta \Delta y\left(\Delta x=x-x_{0}\right.$, $\left.\Delta y=y-y_{0}\right)$ con $\alpha, \beta$ costanti, tale che:

$$
\lim \frac{1}{P_{0} P}\left[f(P)-f\left(P_{0}\right)-\alpha \Delta x-\beta \Delta y\right]=0,
$$

quando $P \rightarrow P_{0}$ in modo che $\lim P_{v} P$ sia uua semitangente determinata, ma qualunque di $I$ in $P_{0}$.

Dal punto di vista lato, che è quello di Storz, $\alpha \Delta x+\beta \Delta y$ è un differenziale totale di $f(P)$ in $P_{r}$, quando la $(3)$ vale comunque $P$ tenda a $P_{0}$.

Orbene, sussiste il teorema: STrotz.

Se $f(P)$ è differenziabile in $P_{0}$ in senso ristretto, lo è pure nel senso di

Supponiamo che la (3) valga in senso ristretto e indichiamo con $\bar{P}(x, y, z)$, $\bar{P}_{0}\left(x_{0}, y_{0}, z_{0}\right)$ i punti del diagramma $F$, di equazione $z=f(P)$, corrispondenti ai punti $P(x, y), P_{0}\left(x_{0}, y_{0}\right)$ di $I$.

Se $P \rightarrow P_{0}$ in $I$ in modo che $\lim P_{0} P=\lambda$, la (3) porge senz' altro (n. 4):

$$
\lim \frac{f(P)-f\left(P_{0}\right)}{P_{0} P}=\lim \frac{\varphi(P)-\varphi\left(P_{0}\right)}{P_{0} P} .
$$

Pertanto la semiretta $\bar{P}_{0} \bar{P}$ ha un limite determinato $\bar{\lambda}$, che giace in (1); ciò̀ le tangenti in $\bar{P}_{0}$ al diagramma $F$ giaccion tutte in un piano, che perciò si dirà il piano tangente in $P_{0}$ ad $F$. 
Proviamo che I'angolo della semiretta $\bar{P}_{0} \bar{P}$ col piano (1) ha sempre per limite zero, comunque $P$ tenda a $P_{0}$ e ciò anche se la semiretta $P_{0} P$ e quindi la $\widetilde{P}_{0} \bar{P}$ non hanno limite. Questa può, grosso modo, ritenersi conseguenza ovvia dal fatto che il piano (1) contiene tutte le semirette di accumulazione comuni agl' insiemi descritti da $P_{0} P$, per $P \rightarrow P_{0}$.

Volendo precisare si ragionerà così. Se, scelto arbitrariamente piccolo un angolo $\delta$, può sempre determinarsi un $\varepsilon>0$, tale che nel campo $\Gamma_{\delta}$ limitato dal cono rotondo (a due falde) di apertura $\frac{\pi}{2}-\delta$, avente per asse la perpendicolare in $\vec{P}_{0}$ al piano (1), cada un numero fnito $>0$ di semirette proiettanti da $\bar{P}_{n}$ punti di $F$, distinti da $\bar{P}_{0}$, situati nell'intorno di raggio $\varepsilon$ di $\bar{P}_{0}$, impiecolendo ancora $\varepsilon$, si otterrà che nessuna di quelle semirette cada in $\Gamma_{\delta}$ e pertanto il limite dell' angolo che $\bar{P}_{1} \bar{P}$ forma con (1) sarà zero. Se dunque questo limite non esiste o non è zero, per $\delta$ abbastanza piccolo non c'è aleun $\varepsilon$ soddisfacente alla predetta condizione.

Vuol dire che, per $\delta$ abbastanza piccolo, l'insieme $K_{z}$ delle semirette proiettanti da $\bar{P}_{0}$ punti di $F$, distinti da $\bar{P}_{0}$, situati in $\Gamma_{\delta}$ e nell' intorno di raggio $\varepsilon$, contiene, qualunque sia $\varepsilon$, infinite semirette. Esiste perciò (j) qualche semiretta di $\Gamma_{\delta}$, che è di rccumulazione per $K_{\varepsilon}$, qualunque sia $\varepsilon$. Una tal semiretta è semitingente ad $F$ in $\bar{P}_{n}$ e non giace sul piano (1), contrariainente alla precedente conclusione. E dunque assurdo ammettere che l'angolo formato da $\bar{P}_{0} \bar{P}$ con (1) non abbia per limite zero.

Ciò premesso, indichiamo con $P^{\prime}$ la proiezione di $P$ (e di $\left.\bar{P}\right)$ sul piano (1), secondo $z$. Sussistono allora in valore e segno (comunque scelgasi il verso positivo sul piano $\left.P P_{0} \bar{P} \bar{P}_{0} P^{\prime}\right)$, le relazioni :

$$
\frac{f(P)-f\left(P_{0}\right)}{P_{0} P}=\frac{\sin \vec{r} r}{\sin z \overrightarrow{r^{r}}}, \quad \frac{\varphi(P)-\varphi\left(P_{n}\right)}{P_{4} P}=\frac{\sin r^{\prime} r}{\sin z r^{\prime}},
$$

ove $r, \bar{r}, r^{\prime}$ denotan le semirette $P_{0} P, \bar{P}_{0} \bar{P}, \bar{P}_{0} P^{\prime}$. Dunque:

$$
H(P)=\frac{1}{P_{0} P}\left[f(P)-f\left(P_{0}\right)-\alpha \Delta x-\beta \Delta y\right]=\frac{\sin \bar{r} r \sin z r^{\prime}-\sin z \bar{r} \sin r^{\prime} r}{\sin z \bar{r} \sin z r^{\prime}},
$$

da cui, mercè il legame fra i seni di quattro direzioni orientate di un piano, si trae:

$$
H(P)=\frac{\sin \bar{r} r^{\prime} \sin z r}{\sin z \bar{r} \sin \approx r^{\prime}}
$$

(i) Ved. la mia Nota di Cracovia, n. 4. 
Si determini ora un cono $\Gamma_{\delta}$, come quello sopra definito, che contenga nel suo interno la parallela per $\bar{P}_{0}$ all' asse $z$; e sia $\theta$ il minimo angolo formato da $z$ colle generatrici di questo cono $(\theta>0)$, $\psi$ l'angolo $>0$, che $z$ forma con (1). Siccome l'angolo di $\bar{r}$ con (1) tende a zero, per ogni $P$ di un intorno abbastanza ristretto di $P_{0}$ varranno le disuguaglianze:

e quindi :

$$
|\sin z \bar{r}| \geq \sin \theta, \quad\left|\sin z r^{\prime}\right| \geq \sin \psi
$$

$$
|H(P)| \leq \frac{\mid \sin \bar{r} r^{\prime}}{\sin z r \mid} \sin \psi
$$

Tendendo comunque $P$ a $P_{0}$ l'angolo acnto $r r^{\prime}$ tende a zero e sin $z r$ si conserva limitato. Perciò $\lim H(P)=0$ e $\alpha \Delta x+\beta \Delta y$ è differenziale in senso lato.

La conclusione è che differenziabilità in senso stretto ed in senso lato si equivalgono; onde si può parlare semplicemente di differenziabilità.

Ossenvazionte. - Se le tangenti al diagramma $F$ in $\bar{P}_{0}$ giacciono in un piano (1) non parallelo all' asse $z$, sarà $\alpha \Delta x+\beta \Delta y$ differenziale di $f$ in senso stretto epperò in senso lato.

Viceversa, se $\alpha \Delta x+\beta \Delta y$ è differenziale di $f$ in $P_{n}$, è evidente che le tangenti di $F$ in $\bar{P}_{0}$ giacciono in (1). Onde $f(P)$ è differenziabile in $P_{0}$ allora e solo allora che esista qualche piano non parallelo a $z$, che contenga le tangenti in $\bar{P}_{0}$ al diagramma $F$; e questo è appunto il risultato del GUARESCH. Il differenziale è unico se in $\bar{P}_{0} e^{\prime}$ è un sol piano tangente a $F$ (non parallelo all' asse z); vi è invece in $P_{0}$ un fascio lineare di differenziali, se $F$ ammette in $\bar{P}_{0}$ una sola tangente.

6. Può darsi che l'espressione:

$$
H(P, Q)=\frac{1}{Q P}[f(P)-f(Q)-\alpha \Delta x-\beta \Delta y],
$$

ove $P, Q$ sieno due punti variabili in $I$ e $\Delta x, \Delta y$ rappresentino le componenti del vettore $Q P$, tenda a zero, comunque $P, Q$ tendano a $P_{0}$. Si dirà allora che $\alpha \Delta x+\beta \Delta y$ è un iperdifferenziale di $f(P)$ in $P_{0}$.

P. es. quando $I$ ̀̀ un dominio piano e $f(P)$ ammette in ogni punto $P_{0}$, interno ad $I$, derivate pariali continue $f_{x}{ }^{\prime}, f_{y}^{\prime}$, si riconosce ovviamente che il differenziale totale di $f$ in $P_{0}$ è iperdifferenziale. Vedremo nel n. 19 che vale pure la proprietà reciproca.

Osservazione. - È evidente che se $\alpha \Lambda x+\beta \Delta y$ è un differenziale o un iperdifferenziale di $f(P)$ in $P_{0}, l \dot{\prime} f(P)$ è differenziabile o iperdifferenziabile 
rispetto a due altri assi carlesiani qualunque $x^{\prime}, y^{\prime}$. Basta per passare dall'uno all'altro differenziale esprimere $\Delta x, \Delta y$ per $\Delta x^{\prime}, \Delta y^{\prime}$.

7. Passiamo a introdurre il concetto intrinseco di derivazione per la funzione $f(P)$, finita nell'insieme serrato $I$, avente in $P_{0}$ un'accumulazione. $\mathrm{e}$ continua in $P_{0}$.

Denotino $P, Q$ due punti variabili in $I$. Consideriamo i rapporti incrementali

$$
\begin{aligned}
& \frac{f(P)-f\left(P_{0}\right)}{P_{0} P}, \\
& \frac{f(P)-f(Q)}{Q P},
\end{aligned}
$$

in cui i denominatori si assumon sempre positivi (sicchè le rette $P_{0} P, Q P$ son rispettivamente orientate da $P_{0}$ a $P$ e da $Q$ a $P$. Se $P \rightarrow P_{0}$ in modo che $\lim P_{0} P=\lambda$, ovvero $P, Q$ tendono a $P_{0}$ in modo ohe $\lim Q P=\lambda$; si potranno cercare, se esîstono finiti, i limiti dei predetti rapporti.

Quando esiste finito il limite del rapporto (6), questo limite si chiamerà la derivata direzionale di f secondo la semitangente $\lambda$ in $P_{0}$ all'insieme $I$ e si designerà con $f_{\lambda}^{\prime}\left(P_{0}\right)$. S' intende naturalmente che questo limite dipenda soltanto da $\lambda$ e non dal modo come $P$ tende a $P_{0}$, sotto la condizione lim $P_{0} P=\lambda$.

Quando esiste finito il limite del rapporto (7), ed è indipendente dal modo come $P, Q$ tendono a $P_{0}$, sotto la condizione $\lim Q P=\lambda$, si dixà che il limite stesso è la iperderivata direzionale di f secondo la semicorda impropria $\lambda$ in $P_{0}$ all' insieme $I$ e si designerà con $f_{\lambda}^{*}\left(P_{0}\right)$. In particolare, quando $\lambda$ sia semitangente, esistendo $f_{\lambda}^{*}\left(P_{0}\right)$ esisterà anche $f_{\lambda}^{\prime}\left(P_{0}\right)$ (ma non viceversa) e sarà $f_{\lambda}^{\prime}\left(P_{0}\right)=f_{\lambda}^{*}\left(P_{0}\right)$

Se, sempre nell' ipotesi che $\lambda$ sia una semicorda impropria, esiste finito. il limite del rapporto (7), allorchè $P, Q$ tendono a $P_{0}$ comunque, compatibilmente colle condizioni che la semiretta $Q P$ tenda a $\lambda$ e le semirette $P_{0} P, P_{0} Q$ tendano a limiti determinati e distinti, ma qualsiansi, quel limite si dirà la ultraderivala direzionale di $f$ secondo la semicorda impropria $\lambda$ in $P_{0}$ all' insieme $I$ e s'indicherà con $f_{\lambda}\left(P_{0}\right)$.

In particolare, quando $\lambda$ sia una semitangente s'intende che la succes. sione descritta da $Q$ possa esser sostituita dal punto fisso $P_{n}$, nel qual caso $\lim Q P=\lim P_{0} P$ coincide con $\lambda$ e $\lim P_{0} Q$ è una semiretta qualunque distinta da $\lambda$.

È chiaro che, se in $P_{11}$ l'insieme $I$ presenta un'accumulazione regolare e se esiste $f_{\lambda}{ }^{*}\left(P_{0}\right)$, esiste $f_{\lambda}\left(P_{0}\right)$ ed è $f_{\lambda}\left(P_{0}\right)=f_{\lambda}{ }^{*}\left(P_{0}\right)$. Quando $\lambda$ sia una semitangente ed esiste $f_{\lambda}\left(P_{0}\right)$, esiste anche $f_{\lambda}^{\prime}\left(P_{0}\right)$ ed ¿̀ $f_{\lambda}^{\prime}\left(P_{0}\right)=f_{\lambda}\left(P_{0}\right)$. 
Cangiando $\lambda$ nell' opposta $-\lambda$, se esiste $f_{\lambda}^{*}\left(P_{0}\right)$ od $f_{\lambda}\left(P_{0}\right)$ esiste pure $f_{-\lambda}{ }^{*}\left(P_{0}\right)$ ed $f_{-\lambda}\left(P_{0}\right)$, e risulta :

$$
f_{\lambda} *\left(P_{0}\right)=-f_{-\lambda}^{*}\left(P_{0}\right), \quad f_{\lambda}\left(P_{0}\right)=-f_{-\lambda}\left(P_{0}\right) .
$$

Se $I$ è un dominio piano e $P_{0}$ un punto ad esso interno, le ordinarie derivate parziali coincidono colle derivate direzionali secondo le direzioni positive $x, y$, mentre le derivate direzionali secondo $-x,-y$ hanno segni contrarì.

Si costruiscono facilmente esempi di funzioni che posseggono derivate dire. zionali in tutle le direzioni uscenti da un punto, ma non ultraderivate; ed esempi di funzioni che posseggono ultraderivate (e quindi derivate) in tutte le direzioni, ma non iperderivate.

P. es. la funzione $z=\sqrt{x^{2}+y^{2}}$ (ove il radicale è positivo) nel punto $x=y=0$ è derivabile in tutte le direzioni, mentre non è parzialmente derivabile (perchè sull' asse $x$ dà luogo ad una funzione che ha in $x=0$ la derivata destra uguale ad 1 e la sinistra uguale a -1 ; e analogamente su $y$ ) e non esiste alcuna direzione in cui sia ultraderivabile (nè, quindi, iperderivabile); una funzione $z=f(x, y)$, differenziabile in ogni punto interno $P_{0}$ di un dominio piano, ma colle derivate parziali non ambedue continue in $P_{0}$, è ultraderivabile in tutte le direzioni (n. 8), ma non iperderivabile (n. 19); una funzione $z=f(x, y)$ colle derivate parziali continue in ogni punto interno $P_{0}$ di un dominio piano, è iperderivabile in tutte le direzioni.

\section{Condizione per la differenziabilità.}

8. Vale in proposito il teorema seguente:

Sia $f(P)$ una funzione finita di un punto $P$ variabile in un insieme piano serrato $I$, avente in $P_{0}$ un' accumulazione regolare. $L a f(P)$ sia continua in $P_{0}$. Allora condizione necessaria e sufficiente perchè $f(P)$ sia differenziabile in $P_{0}$ è che esistano ivi le ultraderivate secondo le semitangenti dell' insieme $I$ in $P_{0}$. In tal caso il differenziale $d i \cdot f(P)$ in $P_{0}$ è individualo.

Assumansi come semiassi cartesiani positivi $x, y$ due semitangenti non allineate di $I$ in $P_{0}$. Se $f(P)$ è differenziabile rispetto a due assi distinti da $x, y$, lo è pure (n. 6, Oss.) rispetto ad $x, y$. Sia $\alpha \Delta x+\beta \Delta y$ un differenziale di $f(P)$ in $P_{0}$. Dalla relazione (n. 5) $\lim H(P)=0$, supposto $\lim P_{0} P=\lambda$, si ricava ovviamente, qualunque siano $\mathrm{i}$ versi delle componenti di $\lambda$ sugli assi $x, y$ :

$$
\lim \frac{f(P)-f\left(P_{n}\right)}{P_{0} P}=\alpha \frac{\sin \lambda y}{\sin x y}+\beta \frac{\sin x \lambda}{\sin x y} .
$$


Ciò premesso, dicasi $\mu$ una semicorda impropria di $I$ in $P_{0}$. Essendo $P_{0}$ regolare, si puó supporre che $P, Q$ variino in $I$ in modo che $\lim P_{0} P, \lim P_{0} Q$ sieno due semitangenti non allineate $\lambda_{1}, \lambda_{2}$ in $P_{0}$ (di cui nna arbitraria) e $\lim Q P=\mu$. Dalla relazione

$$
\frac{f(P)-f(Q)}{Q P}=\frac{f(P)-f\left(P_{0}\right)}{P_{0} P} \frac{P_{0} P}{Q P}+\frac{f(Q)-f\left(P_{0}\right)}{P_{0} Q} \frac{Q P_{0}}{Q P}
$$

(nella quale, si ricordi, $Q P, P_{0} P, P_{0} Q$ son positivi, così che $Q P_{0}$ è negativo), tenendo conto sia delle relazioni derivanti dalla (8) per $\lambda=\lambda_{1}, \lambda_{2}$, sia delle

$$
\lim \frac{P_{0} P}{Q P}=\frac{\sin \mu \lambda_{2}}{\sin \lambda_{1} \lambda_{2}}, \quad \lim \frac{Q P}{Q P}=\frac{\sin \lambda_{1} \mu}{\sin \lambda_{1} \lambda_{2}}
$$

e infine dal legame fra i seni di quattro direzioni orientate di un piano, si deduce che:

$$
\lim \frac{f(P)-f(Q)}{Q P}=\alpha \frac{\sin \mu y}{\sin x y}+\beta \frac{\sin }{\sin \frac{x \mu}{x y}} .
$$

Dunque il primo membro della (10) è indipendente dalla coppia $\lambda_{1}, \lambda_{2}$ e coincide col primo membro della (8) quando $\mu=\lambda$ è una semitangente di $I$ in $P_{0}$ e all' insieme dei $Q$ è sostituito il punto fisso $P_{0}$. Epperò, secondo la definizione del n. 7 , esiste $f_{\mu}\left(P_{0}\right)$. In particolare per $\mu=x, y$ si ha:

$$
f_{x}\left(P_{0}\right)=\alpha, \quad f_{y}\left(P_{0}\right)=\beta ;
$$

e pertanto $\alpha \Delta x+\beta \Delta y$ è individuato. Di più eangiando $\mu$ in $-\mu \operatorname{vien} \theta$ $f_{-\mu}\left(P_{0}\right)=-f_{\mu}\left(P_{0}\right)$.

Viceversa, suppongasi la $f(P)$ ultraderivabile secondo ogni semitangente $\mu$ di $I$ in $P_{0}$. Per la regolarità di $P_{0}, \mu$ può ottenersi come limite d' una semiretta $Q P(Q, P$ variabili in $I)$ per $Q, P$ tendenti a $P_{0}$ in modo che $\lim P_{0} P=x$, $\lim P_{v} Q=y$, ove $x, y$ son due convenienti semitangenti (non allineate) di $I$ in $P_{0}$ (di eui una arbitraria). Dalla $(9)$ in virtù delle

$$
\lim \frac{P_{0} P}{Q P}=\frac{\sin \mu y}{\sin x y}, \quad \lim \frac{Q P_{0}}{Q P}=\frac{\sin x \mu}{\sin x y}
$$

e dal fatto che i rapporti $\frac{f(P)-f\left(P_{0}\right)}{P_{0} P}, \frac{f(Q)-f\left(P_{0}\right)}{P_{0} Q}$ hanno i limiti (11), si deduce la $(10)$. E poichè il valore $f_{\mu}\left(P_{n}\right)$ del primo membro della (10), uguaglia (n. 7) $f_{\mu}^{\prime}\left(P_{0}\right)$, si conclude che tutte le semitangenti ad $F$ in $\bar{P}_{0}$ giaccion sul piano (1). Dunque $\alpha \Delta x+\beta \Delta y$ è differenziale di $f$ in $P_{0}$. 
9. Se $I$ possiede in $P_{0}$ una sola semitangente $\lambda$, condiaione necessaria $e$ sufficiente per la differenziabilita di $f(P)$ in $P_{0}$, è che esiste la derivata $f_{\lambda}^{\prime}\left(P_{0}\right)$; se I possiede in $P_{0}$ due semitangenti opposte $\lambda,-\lambda$, condizione necessaria $e$ sufficiente per la differenziabilitì, è che esistano $f_{\lambda}^{\prime}\left(P_{0}\right), f_{-\lambda}^{\prime}\left(P_{0}\right)$ ed abbian valori opposti. In questi casi si ha in $P_{0}$ un fascio lineare di differenziali.

Questo teorema è conseguenza ovvia dell' equivalenza del fatto analitico che esistano $\infty^{1}$ differenziali in $P_{0}$ e del fatto geometrico che $F$ possegga in $\bar{P}_{0}$ una sola tangente (n. 5, Oss.). Quando esistano $f_{\lambda}^{\prime}\left(P_{0}\right), f_{-\lambda}^{\prime}\left(P_{0}\right)$ ed abbian valori non opposti, il diagramma $F$ possiede in $P_{0}$ un sol piano tangente, ma questo è parallelo all'asse z.

10. In particolare, se $I$ è un dominio piano contornato da una linea di JoRDan chiusa, le rette del piano uscenti da un punto interno o da un punto contorno semplice per la linea (cfr. col n. 18) sono tatte tangenti al dominio nel punto stesso, che è un punto d'accumulazione regolare. Dunque una funzione $f(x, y)$ finita sopra una cella piana contonata da una linea di Jordan con tutti $i$ punti semplici, è differenziabile in tutta la cella, contorwo incluso, allora e solo allora che sia ultraderivabile dovunque e in ogni direzione.

Se il contorno ha una cuspide, perchè $f$ sia differenziabile anche nella cuspide occorre e basta che esista l'ultraderivata di f secondo la tangente alla cuspide. Però ivi si hanno $\infty^{1}$ differenaiali.

Ogni retta uscente dalla cuspide è in questo caso corda impropria del dominio.

\section{Comdizione per l'iperdifferenziabilità.}

11. Dimostriamo ora il teorema seguente:

Sia $f(P)$ una funzione finita di un punto $P$ mobile in un insiene piano serrato $I$, avente in $P_{0}$ un' accumulazione. La $f(P)$ sia continua in $P_{0}$. Allora condizione necessaria e sufficiente perchè $f(P)$ sia iperdifferenaiabile in $P_{0}$, ̀̀ che le corde improprie del diagramma $F[z=f(P)]$ nel punto $\bar{P}_{0}$ corrispondente a $P_{0}$, giacciano tulte in (almeno) un piano non parallelo all'asse $z$.

Sia anzitutto $\lim H(P, Q)=0$, comunque $P, Q$ tendano a $P_{0}$ (n. 6). Denotati con $\bar{P}, \bar{Q}$ i punti di $F$ corrispondenti a $P, Q$; con $P^{\prime}, Q^{\prime}$ le proiezioni secondo $\approx$ dei punti $P, Q$ (e $\bar{P}, \bar{Q})$ sul piano (1); e infine con $r, \bar{r}_{;} r^{\prime}$ le semirette $Q P, \bar{Q} \bar{P}, Q^{\prime} P^{\prime}$, si ottiene (come nel n. 5) la relazione:

$$
H(P, Q)=\frac{\sin \bar{r} r^{\prime} \sin z r}{\sin z \bar{r} \sin z r^{\prime}}
$$


da cui segue:

$$
|H(P, Q)| \geq\left|\sin \bar{r} r^{\prime} \sin z r\right| \text {. }
$$

Onde, chiamato $\tau$ l'angolo di $z$ col piano $x y$ :

$$
|H(P, Q)| \geq\left|\sin \bar{r} r^{\prime}\right| \sin \tau
$$

dalla quale si deduce che $\lim \bar{r} r^{\prime}=0$; epperò le corde improprie di $F$ giaccion tutte nel piano (1).

Viceversa, se il piano (1) contiene tutte le corde improprie di $F$ in $\bar{P}_{0}$, l'angolo di $\bar{r}$ con (1) tende a zern comunque $P, Q$ tendano a $P_{0}$. La cosa è pressochè evidente; ma, se si vuol precisarla, basterà ripetere qui un ragionamento perfettamente analogo a quello svolto, in condizioni simili, per le semitangenti, nel n. 5. Si posson pertanto definire gli angoli $\theta$, $\psi$, analogamente a quanto fu fatto nel n. 5 ; e si ottiene così:

$$
|H(P, Q)| \leq \frac{\left|\sin \bar{r} r^{\prime} \sin z r\right|}{\sin \theta \sin \psi}
$$

Ora $\sin \bar{r} r^{\prime}$ tende a zero e $\sin z r$ si conserva limitato; dunque $\lim H(P, Q)=0$, comunque $P, Q$ tendano a $P_{0}$ ed $f(P)$ ə̀ perciò iperdifferenziabile in $P_{0}$.

Osservazione. - L'iperdifferenziale di $f$ in $P_{0}$ sarà individuato se in $\bar{P}_{0}$ il diagramma $F$ possiede almeno due corde improprie non allineate e non situate in un piano parallelo a $z$; potrà variare in un fascio, se in $P_{0}$ vi è una sola corda impropria.

12. Se $P_{0}$ è un punto d' accumulazione regolare per $I$, condizione necessaria e sufficiente per l'iperdifferenziabilità di $f(P)$ in $P_{0}$ è che la funzione sia iperderivabile secondo ogni semicorda impropria di $I$ in $P_{0}$. In tal caso l'iperdifferenziale è individuato.

Sia $\alpha \Delta x+\beta \Delta y$ un iperdifferenziale di $F$ in $P_{0}$. Allora (n. 11) tutte le corde improprie di $F$ in $\bar{P}_{0}$ giaccion sul piano (1). E siccome fra queste corde, attesa lá regolarità di $P_{0}$, vi son due tangenti distinte, così intanto si conclude che $\alpha \Delta x+\beta \Delta y$ è individuato.

Ciò posto, designamo con $\lambda$ una semicorda impropria di $I$ in $P_{0}$ e con $P, Q$ due punti di $I$, che tendan comunque a $P_{6}$, sotto la sola condi. zione $\lim Q P=\lambda$. Indichiamo inoltre con $H_{\varepsilon}$ l'insieme delle semirette $Q P$, i cui $Q, P$ giacciono in un intorno di raggio $\varepsilon$ di $P_{0}$ e con $\bar{H}_{\mathrm{s}}$ l'insieme delle corrispondenti semirette $\bar{Q} \bar{P}$. Ogni semiretta $\bar{\lambda}$, che sia di accumulazione per $\bar{H}_{\Xi}$ per $\varepsilon$ qualunque, ha per proiezione $\lambda$, perchè questa è l'unica retta 
di accumulazione comune agli $H_{\dot{\varepsilon}}$. $\mathrm{E}$ siccome $\bar{\lambda}$, in quanto semicorda im. propria di $F$, giace sul piano (1), ne segue che $\bar{\lambda}$ è individuata come intersezione del piano (1) col piano parallelo a $z$ condotto per $\lambda$. Ciò significa che il rapporto incrementale (7) tende verso un limite determinato e finito espresso da (n. 4):

$$
f_{\lambda}^{*}\left(P_{0}\right)=\alpha \frac{\sin \lambda y}{\sin x y}+\beta \frac{\sin x \lambda}{\sin x y}
$$

Proviamo che la condizione enunciata è sufficiente. Bisogna dimostrare che ogni corda impropria di $F$ in $\bar{P}_{0}$ sta sul piano (1), perchè ciò equivale appunto (n. 11) ad affermare che $f(P)$ è iperdifferenziabile in $P_{0}$.

Sia $\lambda$ una qualsiasi semicorda impropria di $I$ in $P_{0}$. Attesa la regolarità di $P_{0}, \lambda$ si può ottenere come limite di una semiretta $Q P(Q, P$ variabili in $I$ ) per $Q, P$ tendenti a $P_{0}$ in modo che $\lim P_{0} P=x, \lim P_{0} Q=y$, ove $x, y$ son due semitangenti convenienti non allineate (di eui una arbitraria). Per ipotesi esistono $f_{x}^{*}\left(P_{0}\right)=\alpha, f_{y}^{*}\left(P_{0}\right)=\beta$. Dalla relazione $(9)$, tenuto conto che

$$
\lim \frac{P_{0} P}{Q P}=\frac{\sin \lambda y}{\sin x y}, \quad \lim \frac{Q P_{0}}{Q P}=\frac{\sin x \lambda}{\sin x y}
$$

e che:

$$
\lim \frac{f(P)-f\left(P_{0}\right)}{P_{0} P}=\alpha, \quad \lim \frac{f(Q)-f\left(P_{0}\right)}{P_{0} Q}=\beta
$$

si ricava:

$$
\lim \frac{f(P)-f(Q)}{Q P}=\alpha \frac{\sin \lambda y}{\sin x y}+\beta \frac{\sin x \lambda}{\sin x y}
$$

Ma siccome, esistendo la iperderivata $f_{\lambda} *\left(P_{0}\right)$, il limite del rapporto $\frac{f(P)-f(Q)}{Q P}$ resta lo stesso comunque $P, Q$ tendano a $P_{n}$, purchè lim $Q P=\lambda$, la precedente relazione riducesi alla (12), la quale esprime che ad ogni semicorda impropria $\lambda$ di $I$ in $P_{0}$ corrisponde una semicorda impropria di $F$ in $\bar{P}_{0}$, avente per proiezione $\lambda$ e situata sul piano (1). Poichè, viceversa, ogni semicorda impropria di $F$ in $\bar{P}_{0}$ si proietta in una semicorda di $I$ in $P_{0}$, si conclude che tutte le corde improprie di $F$ in $\bar{P}_{0}$ giaccion sul piano (1); c. d. d.

13. Se $I$ ammette in $P_{0}$ una sola corda impropria, condizione necessaria e sufficiente per la iperdifferenziabilitì di $f(P)$ in $P_{0}$ è che $f(P)$ sia iperderivabile secondo una (e quindi secondo l'altra) delle due semicorde in cui $P_{0}$ divide quella corda. In tal caso vi è in $P_{0}$ un fascio di iperdifferenziali. 
Infatti, quando $I$ ha in $P_{v}$ una sola corda impropria $b$, il diagramma $F$ non può avere che nna sola corda impropria in $\bar{P}_{0}$ o più corde improprie situate sul piano parallelo a $\approx$ condotto per $b$. Se $f(P)$ è iperdifferenziabile in $P_{0}$ quest' ultima eventualità va esclusa (n. 11). Ma allora ogni piano (1) passante per l'unica corda impropria di $F$ in $P_{0}$ (e non parallelo all'asse $z$ ) dà.luogo ad un iperdifferenziale (n. 11).

14. Un esempio di una $f(P)$ possedente in $P_{0}$ un fascio di iperdifferenziali è dato dalla $z=f(P)$ di un punto $\bar{P}$ mobile su una linea di JoRDAN $F$, che si proietti biunivocamente sulla linea $I$ del piano $x y$ e che abbra nel punto $\bar{P}_{0}$ un punto semplice. Invero, in $\bar{P}_{0}$ il diagramma $F$ possiede una sola corda impropria: la tangente.

Ma una $f(P)$ può anche possedere in $P_{0}$ un fascio di differenziali $e$ un solo iperdifferenziale.

Un esempio elegante è fornito dalla $z=f(P)$ di un punto $\bar{P}$ mobile sopra un diagramma $F$ costituito da due linee di JoRDAN $l, m$ tracciate sopra una superficie di JORDAN $\Phi$, nelle ipotesi che le $l, m$ si proiettin biunivocamente sul piano $x y$; che $l, m$ passino semplicemente per $\bar{P}_{0}$, toccandosi ivi; che $\Phi$ abbia in $\bar{P}_{0}$ an punto semplice col piano tangente $\pi$ non parallelo a $z$.

In questo caso le corde improprie al diagramma $F$ in $\ddot{P}_{0}$ riempiono il fascio $\bar{P}_{0}$ sopra $\pi$. A $\pi$ corrisponde il solo iperdifferenziale che $f(P)$ ha in $P_{0}$. Agli altri piani passanti per la tangente comune corrispondono altrettanti differenziali.

La stessa conclusione vale se $l, m$ son rami analitici lineari di origine $\bar{P}_{0}$. Allora il piano delle corde improprie di $F$ in $\bar{P}_{0}$ ò il cosidetto piano principale di HadPHeN, luogo dei punti da cui i rami $l, m$ si proiettano sopra un piano secondo due rami aventi un contatto più intimo di quello che hanno le proiezioni da un punto generico.

Se il piano $\pi$ (tangente a $\Phi$ o piano principale dei due rami) è parallelo a $z$, ma la tangente comune non lo è, si ha l'esempio di una $f(P)$ che possiede in $P_{0}$ un fascio di differenziali e nessun iperdifferenziale.

\section{La regola generale di derivazione delle funzioni composte.}

15. Le considerazioni precedenti permettono di decidere quand'è che ad una $f(P)$ può applicarsi la regola di derivazione delle funzioni composte, in tesa nella più generale accezione. Si dirà che tale regola è applicabile, quando, 
fissata una qualunque semitangente $\lambda$ di $I$ in $P_{g}$ e considerato $u n$ sottoinsieme $K$ di $I$ avente in $P_{0}$ la sola semitangente $\lambda$, sussiste la relazione:

$$
g_{\lambda}{ }^{\prime}\left(P_{0}\right)=\alpha x_{\lambda}{ }^{\prime}\left(P_{0}\right)+\beta y_{\lambda}{ }^{\prime}\left(P_{0}\right),
$$

ove $g(P)$ è la fanzione $f(P)$ per $P$ variabile in $K ; \alpha, \beta$ son due coefficienti numerici, indipendenti dal sottoinsieme $K$ considerato, e $x_{2}^{\prime}\left(P_{0}\right), y_{\lambda}^{\prime}\left(P_{0}\right)$, derivate direzionali secondo $\lambda$ delle coordinate $x, y$ del punto $P$ mobile in $K$, dipendono solo da $\lambda$.

È facile riconoscere che la regola suddetta è applicabile allora e solo allora che $f(P)$ ammetta in $P_{0}$ almeno un differenziale.

Invero, se $\alpha \Delta x+\beta \Delta y$ d un differenziale di $f(P)$ in $P_{0}$, sarà:

$$
g_{\lambda}^{\prime}\left(P_{0}\right)=f_{\lambda}^{\prime}\left(P_{0}\right)=\alpha \frac{\sin \lambda y}{\sin x y}+\beta \frac{\sin x \lambda}{\sin x y},
$$

e d'altronde è chiaro che

$$
x_{\lambda}{ }^{\prime}\left(P_{0}\right)=\frac{\sin \lambda y}{\sin x y}, \quad y_{\lambda}^{\prime}\left(P_{0}\right)=\frac{\sin x \lambda}{\sin x y} .
$$

Viceversa, se la (13) vale comunque sieno scelti $\lambda$ e il sottoinsieme $K$, le tangenti a $F$ in $\bar{P}_{0}$ giaccion sul piano (1), onde (n. 5) $\alpha \Delta x+\beta \Delta y$ è un differenziale di $f(P)$ in $P_{0}$.

Quando $K$ sia un arco rettificabile di origine $P_{0}$, avente ivi la semitangente $\lambda$, risulta in particolare

$$
x_{\lambda}^{\prime}\left(P_{0}\right)=\frac{d x}{d s}, \quad y_{\lambda}^{\prime}\left(P_{0}\right)=\frac{d y}{d s}
$$

ove $s$ sia la lunghezza dell' arco $P_{0} P$ da $P_{0}$ verso $P$.

16. Se gli assi positivi $x, y$, cui riferiscesi il differenziale $\alpha \Delta x+\beta \Delta y$, son due semicorde (in particolare semitangenti) di $I$ in $P_{0}$, e $P_{0}$ è regolare, i coefficienti $\alpha, \beta$, che compaiono nella regola di derivazione delle funzioni composte, son le ultraderivate direzionali di $f$ secondo $x, y$; altrimenti i numeri stessi non hanno significato intrinseco rispetto ad $f(P)$.

Ma, come il differenziale di $f(P)$ in $P_{0}$ si può scrivere, ove esista, rispetto a due assi cartesiani qualunque (n. 6, Oss.), così la regola di derivazione delle funzioni composte può riferirsi a due assi qualunque. Se $x, y$ son due semitangenti non allineate di $f(P)$ in $P_{n}$, risulta (tanto se $P_{0}$ è regolare, quanto se non lo è):

$$
\alpha=f_{x}^{\prime}\left(P_{0}\right), \quad \beta=f_{y}^{\prime}\left(P_{0}\right) .
$$


Presi due assi cartesiani qualunque $x^{\prime}, y^{\prime}$, oltre alla (13), vale la:

$$
g_{\lambda}^{\prime}\left(P_{0}\right)=f_{\lambda}^{\prime}\left(P_{0}\right)=\alpha^{\prime} \frac{\sin \lambda y^{\prime}}{\sin x^{\prime} y^{\prime}}+\beta^{\prime} \frac{\sin x^{\prime} \lambda}{\sin x^{\prime} y^{\prime}},
$$

ove

$$
\alpha^{\prime}=\alpha \frac{\sin x^{\prime} y}{\sin x y}+\beta \frac{\sin x x^{\prime}}{\sin x y}, \quad \beta^{\prime}=\alpha \frac{\sin y^{\prime} y}{\sin x y}+\beta \frac{\sin x y^{\prime}}{\sin x y},
$$

le quali definiscono i coefficienti $\alpha^{\prime}, \beta^{\prime}$ con cui si compone il differenziale totale di $f(P)$ in $P_{0}$, rispetto agli assi $x^{\prime}, y^{\prime}$, allorchè esso è intrinsecamente individuato dalle due derivate direzionali $f_{x}{ }^{\prime}\left(P_{0}\right), f_{y}^{\prime}\left(I_{0}^{\prime}\right)$.

I numeri $\alpha^{\prime}, \beta^{\prime}$ non posson però generalmente concepirsi come limiti di rapporti incrementali. Tuttavia, da un punto di vista puramente formale, $\alpha^{\prime}, \beta^{\prime}$ son da considerarsi come derivate di $f$ in $P_{0}$, rispetto ad $x^{\prime}, y^{\prime}$, in quanto definiscono il differenziale $\alpha^{\prime} \Delta x^{\prime}+\beta^{\prime} \Delta y^{\prime}$. Sono le derivate perfette del GUARESCHI.

Se $f(P)$ ha in $P_{0}$ un fascio di differenziali, ogni coppia $\alpha, \beta$ di numeri soddisfacenti alla (13) puó esser assunta come coppia di derivate perfette di $f$ rispetto a due assi qualunque $x, y$; ma in realtà in questo caso la regola di derivazione delle funzioni composte dice ben poco, perchè l'insieme $I$ e un qualunque suo sottoinsieme $K$ hanno in $P_{0}$ la medesima tangente.

\section{Continuità delle iperderivate quando esistono in un dominio piano.}

17. Cominciamo dal caso delle funzioni di una variabile.

Per una funzione $f(P)$ di un punto $P$ variabile in un insieme rettilineo $I$, si posson definire, in un punto $P_{0}$ d'accumulazione di $I$, le derivate e le iperderivate direzionali (ma non le ultraderivate).

Se l'insieme $I$ è addirittura un intervallo, si riconosce immediatamente che l'iperderivata esiste in ogni punto di $I$ quando ivi esista la derivata ordinaria e sia continua.

Molto più interessante è la proposizione reciproca, che pure è vera. Vale cioè il teorema:

Se una funzione $f(P)$ di un punto $P$ variabile in un intervallo rettilineo $I$, è iperderivabile in ogni punto dell' intervallo, la derivata $f^{\prime}(P)$ è dovunque continua. (La reciproca è ovvia).

Dimostreremo questo teorema nel n. successivo, riducendolo ad un' espressiva proprietà geometrica, che gli equivale. 
18. Conviene che ricordiamo prima la definizione di punto semplice $P$ di una varietà topologica $M_{k}$ appartenente ad uno spazio $S_{n}$, data nella mia Nota di Cracovia.

Il punto $P$ (che può esser interno o situato sul contorno di $M_{k}$ ) dicesi semplice, quando le corde improprie di $M_{k}$ per $P$ giacciono in uno spazio lineare $S_{k}\left({ }^{6}\right)$. Tale definizione equivale a quest' altra: Il punto $P$ è semplice, quando un $S_{n-k}$ prossimo a $P$ sega $M_{k}$ nell'intorno di $P$ al più in un punto (esattamente in un punto se $P$ è interno ad $M_{k}$ ). Fanno eccezione soltanto gli $S_{n-k}$ abbastanza prossimi a spazi $S_{n-k}$ appartenenti ad $u n$ certo $S_{k}$ passante per $P$, che si chiama lo $S_{k}$ tangente ad $M_{k}$ in $P$.

Ebbene, vale il teorema:

Se una linea di Jordan ha tutti $i$ punti semplici, la tangente in un punto su essa mobile varia con continuità ( ${ }^{\mathrm{T}}$ ).

Basta dimostrare il teorema per le linee piane (cfr. col successivo n. 28).

Scelta sul piano una direzione $y$ non parallela alla retta tangente alla linea data $F$ in suo punto $\bar{P}_{0}$, se $\bar{P}_{0}$ è interno ad $F$ le rette abbastanza prossime a $\bar{P}_{0}$, parallele ad $y$, segan la linea attorno a $\bar{P}_{0}$ in un sol punto. Onde $F$ può esser rappresentata intorno a $\bar{P}_{0}$ da un' equazione del tipo $y=f(P)$, ove $P$ varii in un intorno del punto $P_{0}$ proiezione di $\bar{P}_{0}$ sopra un asse $x$.

La conclusione analoga vale se $\bar{P}_{0}$ è un estremo di $F$, purchè si assuma come asse $y$ una retta non tangente ad $F$ in $\bar{P}_{0}$ e si considerino le parallele ad $y$ situate da quella parte di $y$ cui appartiene un intorno abbastanza ristretto di $\bar{P}_{0}$ su $F$. Allora $y=f(P)$ sarà definita soltanto da una parte di $P_{0}$.

Dall'ipotesi che $F$ abbia in $\bar{P}_{0}$ una sola corda impropria (coincidente colla tangente), segue che in $P_{0}$ la $f(P)$ ammette l:iperderivata direzionale (nelle due direzioni uscenti da $P_{0}$, se il punto è interno all'intervallo $I$ dove risulta definita $f(P)$, in una sola direzione, se $P_{0}$ è un estremo di $I$ ). In ogni punto interno di $F$ vi son dunque due semitangenti opposte.

Viceversa, se $f(P)$ è iperderivabile in ogni punto dell' intervallo $I$, il dia.

(6) Nella mia Nota, Le curve intuitive ("Rendiconti di Palermo », 1930) un punto sem. plice $P$ di una linea piana è definito dalla condizione che vi sia in esso una sola tangente sulla quale si possa proiettare ortogonalmente un intorno di $P$ sulla linea. Questa definizione è più generale di quella del testo: ossia un punto $P$ può esser semplice nel senso attuale e non esserlo nel senso della Nota di Cracovia. Però allora la tangente in quel punto ha ivi una discontinuità. Un esempio è fornito da una curva $y=f(x)$, in cui $f(x)$ sia derivabile in un intervallo, con derivata non sempre continua. È chiaro che la definizione della Nota di Cracovia aderisce meglio alla nozione intuitiva di punto semplice.

(7) Ved. il n. 85 del libro eitato di Bouligand ove è dimostrato un teorema equivalente a questo, sul fondamento della semicontinuità superiore del "paratingente». 
gramma $y=f(P)$ ha in ogni punto una sola corda impropria e quindi ogni tal punto è semplice pel diagramma. Da ciò intanto segue l'equivalenza della proprietà geometrica da dimostrarsi e del teorema del n. 17.

Passiamo a stabilire il teorema geometrico. Fissato un punto $P_{0}$ di $I$, diciamo $t_{0}$ la tangente al diagramma $F$ nel punto $P_{0}$ corrispondente e $t$ la tangente nel punto $\bar{P}$ corrispondente a $P$ mobile su $I$ Indichiamo inoltre con $K_{\varepsilon}$ l'insieme delle tangenti a $F$ nei punti situati in un intorno di raggio $\varepsilon$ di $\bar{P}_{0}$. Esistono certamente rette di accumalazione per $K_{\varepsilon}$, qualunque sia $\varepsilon$; sia $r$ una di queste. Occorre dimostrare che $r$ coincide necessariamente con $t_{0}$.

Scelti un numero $\varepsilon>0$ e un angolo (acuto) $\hat{\partial}$, esiste qualche tangente $t$ ad $F$ in un punto distinto da $\bar{P}_{n}$, situato nell'intorno di raggio $\varepsilon$ di $\bar{P}_{0}$, la quale forma con $r$ un angolo $<\delta$. Ragionando come nel n. 4 della mia Nota di Cracovia, si conclude che è possibile trovare in $F$ un insieme numera. bile $\bar{P}_{1}, \bar{P}_{z}, \ldots$ di punti convergente a $\bar{P}_{0}$ e tale che la tangente $t_{n}$ in $\bar{P}_{n}$, per $n \rightarrow \infty$, tenda ad $r$. Da questo dedurremo che $r$ è corda impropria di $F$ in $\bar{P}_{0}$ e quindi che coincide con $t_{0}$.

Si fissino all' uopo due successioni decrescenti e infinitesime di numeri positivi non nulli e di angoli acuti non nulli:

$$
\varepsilon_{1}, \varepsilon_{2}, \ldots ; \delta_{1}, \delta_{2}, \ldots
$$

Sia $\bar{P}_{1}^{\prime}$ il primo punto della successione $\bar{P}_{n}$, che trovasi nell' intorno di raggio $1 / 2 \varepsilon_{1}$ di $\bar{P}_{0}$ e $t_{1}^{\prime}$ la tangente a $F$ in $\bar{P}_{1}^{\prime}$. Considerato l'intorno di raggio $1 / 2 \varepsilon_{1}$ di $\bar{P}_{1}^{\prime}$, ivi potrà seegliersi (pel principio $Z$ del n. 2 della mia Nota di Cracovia) un punto $\bar{P}_{1}^{\prime \prime}$ distinto da $\bar{P}_{1}^{\prime}$, tale che la retta $\bar{P}_{1}^{\prime} \bar{P}_{1}^{\prime \prime}$ formi con $t_{1}^{\prime}$ un angolo acuto $<\delta_{1}$. Sia similmente $\bar{P}_{2}^{\prime}$ il primo punto della successione $\bar{P}_{n}^{\prime}$, distinto da $P_{1}^{\prime}$, che trovasi nell' intorno di raggio $1_{2} \varepsilon_{2}$ di $\bar{P}_{0}$ e $t_{z}^{\prime}$ la tangente ad $F$ in $\bar{P}_{2}^{\prime}$. Si potrà allora determinare nell'intorno di raggio $1_{2} \varepsilon_{2}$ di $\bar{P}_{z}^{\prime}$ un punto $\bar{P}_{2}^{\prime \prime}$, distinto da $\bar{P}_{z}^{\prime}$, tale che la retta $\bar{P}_{2}^{\prime} \bar{P}_{2}^{\prime \prime}$ formi con $t_{2}^{\prime}$ un angolo aeuto $<\delta_{2}$; e così proseguendo.

La retta $\bar{P}_{n}^{\prime} \bar{P}_{n}^{\prime \prime}$ viene in tal guisa ad avere $\mathrm{i}$ suoi punti distinti $\bar{P}_{n}^{\prime}, \bar{P}_{n}^{\prime \prime}$ nell'intorno di raggio $\varepsilon_{n}$ di $\bar{P}_{0}$ e l'angolo acuto (o retto) $\varphi_{n}$, ch'essa forma con $r$ è minore dell' angolo $\delta_{n}$ aumentato dell' angolo acuto (o retto) $\theta_{n}$, che la tangente $t_{n}^{\prime}$ a $F$ in $\bar{P}_{n}^{\prime}$ forma con $r$.

Presi ora ad arbitrio il numero $\varepsilon>0$ e l'angolo acuto $\delta>0$, da un certo $n$ in poi risulta $\varepsilon_{n}<\varepsilon, \delta_{n}<1 / 2 \delta, \theta_{n}<1 / 2 \delta$, onde $\varphi_{n}<\delta$; epperò $\lim _{n \rightarrow \infty} \bar{P}_{n}^{\prime} \bar{P}_{n}^{\prime \prime}=r ;$ c. d. d. 
Osservazione. - Una linea di Jordan a punti semplici risulla sempre rettificabile, appnnto perchè la sua tangente è variabile con continuità, così che è applicabile la conclusione del n. 7 della mia citata Nota sulle curve intuitive. Essa ammette sempre perciò una rappresentazione parametrica regolare in grande.

19. Consideriamo ora una funzione $f(P)$ di un punto $P$ variabile in un dominio piano $I$. Vale il teorema analogo a quello indicato nel. n. 17 per le funzioni di una variabile; cioè:

Condizione necessaria e sufficiente perchè una funzione reale $f(P)$ di un punto variabile in un dominio piano I sia iperdifferenziabile in ogni punto interno al dominio, è che esistano dovunque nell interno di I le derivate par. ziali di $f$ e sieno continue. (La sufficienza della condizione è ovvia).

Questo teorema equivale alla proprietà geometrica, che stabiliremo nel n. successivo.

20. Una superficie di Jordan coi punti tutti semplici ha il piano tangente variabile con continuitì $\left({ }^{8}\right)$.

E chiaro che basterà dimostrare il teorema in piccolo per una superficie dello spazio ordinario.

L'intorno $F$ di un punto $\bar{P}_{0}$ interno alla data superficio può rappresentarsi con un'equazione del tipo $z=f(P)$, ove $f(P)$ sia una funzione reale, continua di un punto $I$ variabile nell'interno di una cella piana $I$, comprendente il punto $P_{0}$ che corrisponde a $\bar{P}_{0}$. Tale rappresentazione si ottiene proiettando l'intorno di $\bar{P}_{0}$ sul piano $x y$, secondo una direzione $z$ non parallela al piano tangente ad $F$ in $\bar{P}_{0}$, tenuto conto della proprietà caratteristica dei punti semplici.

Il fatto che i punti di $F$ sieno semplici (cioè che le corde improprie in ciascuno di essi giacciano sul piano tangente) equivale (n. 11) all'iperdifferenziabilitá della $f(P)$ in ogni punto di $I$ : donde l'asserita equivalenza fra la proprietà geometrica e il teorema del n. 19.

Considereremo dapprima (come del resto nell' enunciato del teor. 19) i punti interni ad $F$, cioè i punti interni ad $I$.

Sia $\lambda$ una semiretta di origine $P_{0}$. Nel punto $\bar{P}$ di $F$, corrispondente a $P$ variabile nell'interno di $I$, vi è una sola tangente $t$ ad $F$, la quale ap-

(8) Ved. a tal proposito il n. 86 del citato libro dî Boulıand. 
partenga alla giacitnra $z \lambda$ dei piani paralleli a $z$ e a $\lambda$. Dimostreremo che la derivata $f_{2}^{\prime}(P)$ è continua in $P_{0}$. Da ciò per $\lambda=x, y$ trarremo la conclu* sione del teorema.

Avvertiamo anzitutto che la continuita di $f_{\lambda}^{\prime}(P)$ in $P_{0}$ equivale al fatto che per $P \rightarrow P_{0}$ la tangente $t$ tende all' analoga tangente $t_{0}$ in $P_{0}$. Invero, se $t$ ha per limite $t_{0}$, quella delle due semitangenti in cui $\bar{P}$ divide $t$, che si proietta nella semiretta avente la direzione $\lambda$, ha per limite l'analoga semi. tangente in $\bar{P}_{0}$.

$D^{\prime}$ ora innanzi indicheremo brevemente con $t$ le tangenti che appartengono alla giacitura $z \lambda$. Scelto un intorno di raggio $\approx$ di $\vec{P}_{n}$, denotiamo con $K_{\varepsilon}$ l'insieme delle $t$ che toccano $F$ nei punti dell'intorno fissato e con $r$ una retta d'accumulazione (uscente da $\bar{P}_{0}$ ) di $K_{\varepsilon}$, qualunque sia $\varepsilon$. Poichè le $t$ appar* tengono alla giacitura $z \lambda$, così sarà di $r$. Per dimostrare il teorema, basterà provare che $r$ è una corda impropria di $F$, perchè allora ne seguirà che $r$ coincide con $t_{0}$, che è l'unica corda impropria di $F$ in $\widehat{P}_{0}$, appartenente alla giacitura $z \lambda$.

Non c'è qui che da ripetere, con lievi cangiamenti di parole, il ragionamento del n. 18. Fissata una successione $\bar{P}_{1}, \bar{P}_{2}, \ldots$ di punti interni ad $F$, che converga a $\bar{P}_{n}$, in modo che $\lim _{n \rightarrow \infty} t_{n}=r$ (ove $t_{n}$ è la $t$ relativa a $\bar{P}_{n}$ ), consideriamo le saccessioni (14). Sia $P_{1}^{\prime}$ il primo dei punti $\bar{P}_{1}, \bar{P}_{2}, \ldots$ ehe cade nell' intorno di raggio $1 / 2 \varepsilon_{1}$ di $\bar{P}_{0}$ e $t_{1}^{\prime}$ la $t$ relativa. Il piano parallelo a $\approx \lambda$ passante per $P_{1}^{\prime}$ taglia $F^{\prime}$ secondo una linea di Jondan, che ha in $P_{1}^{\prime}$ un punto semplice e per tangente ivi $t_{1}^{\prime}$. Si può dunque scegliere nell' intorno di raggio ${ }^{1} / 2 \varepsilon_{1}$ di $\bar{P}_{1}^{\prime}$ un punto $\bar{P}_{1}^{\prime \prime}$ (interno alla linea), distinto da $\bar{P}_{1}^{\prime}$ e tale che la retta $\bar{P}_{1}^{\prime} \bar{P}_{1}^{\prime \prime}$ formi con $t_{1}^{\prime}$ un angolo $<\delta_{1}$; e cosi proseguendo. Si giunge in tal guisa a costruire due successioni $\bar{P}_{1}^{\prime}, \bar{P}_{2}^{\prime}, \ldots ; \bar{P}_{1}^{\prime \prime}, \bar{P}_{2}^{\prime \prime}, \ldots$ di punti interni ad $F$ e tali che $\bar{P}_{n}^{\prime}, \bar{P}_{n}^{\prime \prime}$ giaccion nell'intorno di raggio $\varepsilon_{n}$ di $\bar{P}_{0}$ e la retta $\bar{P}_{n}{ }^{\prime} \bar{P}_{n}{ }^{\prime \prime}$ forma con $t_{n}{ }^{\prime}$ (la $t$ relativa a $\left.\bar{P}_{n}{ }^{\prime}\right)$ un angolo $\psi_{n}<\delta_{n}$. Siccome poi le rette $r, \bar{P}_{n}^{\prime} \bar{P}_{n}{ }^{\prime \prime}, t_{n}^{\prime}$ appartengono alla giacitura $z \lambda$, l'angolo $\varphi_{n}$ che la retta $\bar{P}_{n} \bar{P}_{n}^{\prime \prime}$ forma con $r$ è non maggiore della somma dell' angolo non ottuso $\theta_{n}$ che $t_{n}{ }^{\prime}$ forma con $r$ e dell' angolo $\psi_{n}$; e quindi è minore di $\theta_{n}+\delta_{n}$.

Di qui si deduce (n. 18) che $\lim \bar{P}_{n}^{\prime} \bar{P}_{n}^{\prime \prime}=r$; epperò $r$ è corda im. propria; c.d.d.

Se $P_{0}$ è un punto contorno del dominio piano $I$, la conclusione vale immutata, purchè si riferisca ad una semicorda impropria $\lambda$, di origine $P_{0}$, dell'insieme $I$, tale che per ciascuno dei punti di $I$ situati in un intorno abbastanza piccolo di $P_{0}$ passi una semicorda impropria di $I$ parallela a $\lambda$. Questa 
condizione è sempre soddisfatta pei punti contorno di $I$, quando il contorno è costituito da una linea di Jordar a punti tutti semplici; epperò la continuità del piano tangente ad una superficie di Jordan a punti tutti semplici, vale anche pei punti del contorno, quando questi costituiscono una linea di Jordan a punti semplici.

OSSERVAZIONE. - Nei riguardi della quadrabilitò di una superficie di Jordan $F^{\top}$ a punti tutti semplici è ovvio ch'essa sussiste in piccolo, per ogni cella abbastanza piccola di $F$, la quale sia regolarmente rappresentabile (p. es. per mezzo di una proiezione) sopra una cella piana. La possibilità di una rappresentazione parametrica regolare in grande di una superficie di JoRDAN, che sia una cella a due dimensioni coi punti tutti semplici, non pare sia tanto facile a dimostrarsi.

\section{Condizione per la differenziabilità in un dominio plano.}

21. Stabilita la condizione d'iperdifferenziabilità in un dominio piano, occupiamoci della semplice differenziabilità.

Sia $f(P)$ una funzione definita in un dominio piano $I$ e $P_{v}\left(x_{0}, y_{0}\right)$ sia un punto interno ad $I$. Secondo il n. 8 la condizione necessaria e sufficiente perchè $f(P)$ sia differenziabile in $P_{0}$ (che è un punto d'aecumulazione regolare), è che $f(P)$ sia ultradérivabile secondo ogni direzione nscente da $P_{0}$. Ora questa condizione può rendersi notevolmente più espressiva, nel caso attuale.

Prendiamo come rapporti incrementali parziali (generalizzati) di $f(P)$ in $P_{0}$ i rapporti

$$
\frac{f(x, y)-f\left(x_{0}, y\right)}{x-x_{0}}, \frac{f(x, y)-f\left(x, y_{0}\right)}{y-y_{0}}
$$

nei quali si abbandoni la troppo esigente condizione che il punto $P(x, y)$ si muova rispettivamente sopra una retta $y=$ cost. o rispettivamente $x=$ cost. E soltanto abbandonando tale condizione che si può sperare di far giuocare il vero carattere della $f(P)$, la quale deve concepirsi come funzione della coppia $(x, y)$ e non di ciascuna delle variabili. Ebbene, se comunque $P$ tende a $P_{0}^{\prime}$, sotto la sola condizione che la semiretta $P_{0} P$ tenda ad una semiretta qualsiasi prefissata, in eui non sia rispettivamente costante $x$ od $y$, esistono finiti i limiti dei predetti rapporti, tali limiti si chiameranno le derivate par. ziali generalizzate di $f(P)$ in $P_{0}$ e si designeranno con $f_{\alpha}\left(P_{0}\right), f_{y}\left(P_{0}\right)$.

Sono questi i medesimi simboli delle ultraderivate; ma la cosa non dà 
luogo ad ambiguità, perchè, come risulterà dal seguito, i valori di $f_{x}\left(P_{4}\right), f_{y}\left(P_{0}\right)$, quando esistono entrambi, coincidono appunto coi valori delle ultraderivate.

Stabiliamo il teorema:

Condizione necessaria e sufficiente perchè una funzione $f(P)$ del punto $P(x, y)$ di un dominio piano $I$, sia differenziabile nel punto $P_{0}\left(x_{0}, y_{0}\right)$ interno ad $I$, ̀̀ che in $P_{0}$ esistano le derivate parziali generalizzate $f_{x}\left(P_{n}\right), f_{y}\left(P_{u}\right)$.

La necessità della condizione è già contenuta nel n. 8 , perchè la definizione delle derivate parziali generalizzate fornisce un modo particolare di ottenere, ove esistano, i valori delle ultraderivate omonime.

Stabiliamo la sufficienza della condizione. Se facciamo variare $P(x, y)$ sulla parallela ad $x$ condotta per $P_{4}$, eppoi sulla parallela ad $y$ per $P_{0}$, in forza dell' ipotesi risulta $\alpha=f_{x}\left(P_{0}\right)=\bar{f}_{x}{ }^{\prime}\left(P_{0}\right), \beta=f_{y}\left(P_{0}\right)=\bar{f}_{y}\left(P_{v}\right)$, ove $f_{x}, f_{y}$ denotan le derivate generalizzate e $\bar{f}_{x}{ }^{\prime}, \bar{f}_{y}^{\prime}$ le derivate parziali ordinarie.

Indichiamo con $M\left(x, y_{0}\right), N\left(x_{0}, y\right)$ le proiezioni di $P(x, y)$, comunque variabile, sulle parallele per $P_{0}$ ad $x, y$. Sussiston le relazioni :

$$
\begin{aligned}
& \frac{f(P)-f\left(P_{0}\right)}{P_{0} P}=\frac{f(P)-f(M)}{M P} \frac{M P}{P_{0} P}+\frac{f(M)-f\left(P_{0}\right)}{P_{0} M} \frac{P_{0} M}{P_{0} P} \\
& \frac{f(P)-f\left(P_{0}\right)}{P_{0} P^{\prime}}=\frac{f(P)-f(N)}{N P} \frac{N P}{P_{0} \bar{P}}+\frac{f(N)-f\left(P_{0}\right)}{P_{0} N} \frac{P_{0} N}{P_{0} P} .
\end{aligned}
$$

Supposto che $P \rightarrow P_{0}$ in modo che $\lim P_{0} P=x^{\prime}$ (ove $x^{\prime}$ è equipollente al semiasse positivo $x$ o al semiasse $-x$ ), la semiretta $N P$ tende pure ad $x^{\prime}$. Pertanto viene:

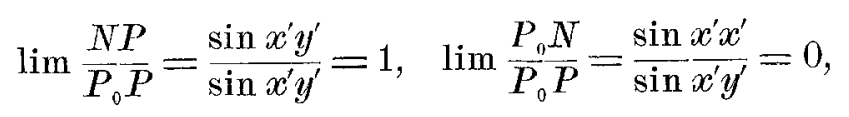

ove $y^{\prime}$ è il limite di $P_{0} N$. La (16) dimostra allora che:

$$
\lim \frac{f(P)-f\left(P_{0}\right)}{P_{0} P}= \pm \alpha
$$

secondo che $x^{\prime}$ è equipollente ad $x$ o a $-x$. Esiste perciò la derivata direzionale $f^{\prime}{ }_{x^{\prime}}\left(P_{0}\right)$. Similmente dalla $(15)$ si deduce che esiste $f_{y^{\prime}}^{\prime}\left(P_{0}\right)= \pm \beta$, valendo il +0 il - secondo che $y^{\prime}$ è equipollente ad $y$ o a $-y$.

Supponiamo ora che $P \rightarrow P_{0}$ in modo che $\lim P_{0} P=\lambda$, ove $\lambda$ è una semiretta non parallela agli assi. Sieno $x^{\prime}, y^{\prime}$ i limiti delle semirette $P_{n} M, M P$. Dalla (15) tenuto conto delle

$$
\lim \frac{M P}{P_{0}} \frac{\sin x^{\prime} \lambda}{\sin x^{\prime} y^{\prime}}, \quad \lim \frac{P_{0} M}{P_{0} P}=\frac{\sin \lambda y^{\prime}}{\sin x^{\prime} y^{\prime}},
$$


si deduce:

$$
\lim \frac{f(P)-f\left(P_{0}\right)}{P_{0} P}=f_{x^{\prime}}^{\prime}\left(P_{0}\right) \frac{\sin \lambda y^{\prime}}{\sin x^{\prime} y^{\prime}}+f_{y^{\prime}}^{\prime}\left(P_{0}\right) \frac{\sin x^{\prime} \lambda}{\sin x^{\prime} y^{\prime}}
$$

cioè :

$$
\lim \frac{f(P)-f\left(P_{0}\right)}{P_{0} P}=\alpha \frac{\sin \lambda y}{\sin x y}+\beta \frac{\sin x \lambda}{\sin x y}
$$

epperò tutte le semitangenti in $\bar{P}_{0}$ alla superficie $F(z=f(P))$, ivi comprese quelle che corrispondono alle direzioni degli assi, giacciono sul piano (1); e quindi (n. 5, Oss.) la $f(P)$ è differenziabile in $P_{0}$.

Osservazione. - Il teorema dimostrato prova che il concetto di derivate parziali generalizzate è quello che più naturalmente estende il concetto di derivata per le funzioni di una variabile, perchè fa equivalere la differenziabilità all' esistenza delle derivate generalizzate, come per le funzioni di una variabile la differenziabilità equivale all' esistenza della derivata.

22. Si può ehiedere se per la differenziabilità di $f(P)$ in un punto $P_{0}$ interno al dominio piano $I$, basti l' esistenza dei limiti dei rapporti incrementali generalizzati sopra le rette uscenti da $P_{0}$. Ebbene, la sola esistenza di questi limiti (finiti) in ogni punto interno ad $I$ nou basta; ma essa divien. sufficiente quando i limiti medesimí, considerati come funzioni del punto $P_{0}$ variabile nell'interno di $I$, siano limitati. Vale cioè il teorema:

Se in ogni punto $(x, y)$ interno ad un dominio piano $I$, dove sia data una funzione $f(x, y)$, esistono, univocamente determinati dal punto, $i$ limiti

$$
\begin{aligned}
& x(x, y)=\lim \frac{f(\bar{x}, \bar{y})-f(x, \bar{y})}{\bar{x}-x} \\
& \beta(x, y)=\lim \frac{f(\bar{x}, \bar{y})-f(\bar{x}, y)}{\bar{y}-y}
\end{aligned}
$$

quando il punto $(\bar{x}, \bar{y})$ tende ad $(x, y)$ sopra una retta qualunque uscente da $(x, y)$, sulla quale non sia rispettivamente costante $x$ od $y$; e le fumzioni $\alpha(x, y), \beta(x, y)$ son limitate nell' interno di $I\left({ }^{9}\right)$, la data funzione $f(x, y)$ è differenziabile nell' interno del dominio.

Anzitutto è chiaro che $f(P)$ ammette in un punto $P_{0}\left(x_{0}, y_{0}\right)$ interno ad $I$

$\left({ }^{9}\right)$ Cioè limitate in ogni dominio interno ad $I$, il che equivale, pel teorema di Weierstrass, a supporre che $x, \beta$ siano limitate nell"intorno di ogni punto interno ad $T$. 
le derivate parziali $\bar{f}_{x}^{\prime}\left(P_{0}\right), \bar{f}_{y}^{\prime}\left(P_{0}\right)$, aventi i valori $\alpha\left(x_{0}, y_{0}\right), \beta\left(x_{0}, y_{11}\right)$. Ne deriva che, se $P, Q$ son due punti distinti variabili nell'interno di $I$ colla condizione che la retta $P Q$ si conservi parallela ad un asse, p. es. $y$, il rapporto $\frac{f(P)-f(Q)}{Q P}$ si mantiene limitato, perchè

$$
|f(P)-f(Q)|=Q P|\beta(R)|,
$$

ove $R$ è un conveniente punto interno al segmento $Q P$.

Proviamo ora che in $P_{0}$ esiston altresi le derivate direzionali secondo gli assi e che:

$$
\begin{aligned}
& \alpha\left(x_{11}, y_{0}\right)=\bar{f}_{x}^{\prime}\left(P_{0}\right)=f_{x}{ }^{\prime}\left(P_{0}\right)=-f_{-x}^{\prime}\left(P_{0}\right) \\
& \beta\left(x_{0}, y_{0}\right)=\bar{f}_{y}^{\prime}\left(P_{0}\right)=f_{y}^{\prime}\left(P_{0}\right)=-f_{-y}^{\prime}\left(P_{0}\right) .
\end{aligned}
$$

Conservando le notazioni del n. prec., si muova dalla (15) e facciasi tendere $P$ a $P_{0}$ in modo affatto arbitrario, colla sola condizione che $\lim P_{0} P=x^{\prime}$ sia una semiretta parallela all' asse $x$ (equipollente ad $x$ o a $-x$ ). Sarà allora $\lim P_{0} M=x^{\prime}$. Si chiami $y^{\prime}$ il limite di $M P$. Viene:

$$
\lim \frac{M P}{P_{0} P}=\frac{\sin x^{\prime} x^{\prime}}{\sin x^{\prime} y^{\prime}}=0, \quad \lim \frac{P_{0} M}{P_{0} P}=\frac{\sin x^{\prime} y^{\prime}}{\sin x^{\prime} y^{\prime}}=1
$$

e, siccome $\frac{f(P)-f(M)}{M P}$ si conserva limitato, perchè la retta $P M$ è sempre parallela ad $y$, così la (15) prova l'esistenza del limite del primo membro, cioè l'esistenza di $f^{\prime}{ }_{x^{\prime}}\left(P_{0}\right)$ e il fatto che:

$$
f_{x^{\prime}}^{\prime}\left(P_{0}\right)=\lim \frac{f(M)-f\left(P_{n}\right)}{P_{0} M}= \pm \alpha\left(x_{0}, y_{0}\right)
$$

valendo il + o il - secondo che $x^{\prime}$ è equipollente ad $x$ o a $-x$.

Similmente dalla $(16)$ si deduce I'esistenza di $f^{\prime}{ }_{y}^{\prime}\left(P_{0}\right)$, ove $y^{\prime}$, parallela ad $y$, sia il limite di $P_{0} P$, e si dimostra la seconda delle (20).

Suppongasi ora che $P(x, y)$ muovasi sopra una semiretta $\lambda$ per $P_{0}$, non parallela agli assi, sicchè rimane fisso il segno di $y-y_{0}$. Quando $y \rightarrow y_{0}$ (cioè $P \rightarrow P_{0}$ ) la seconda delle (19) porge:

$$
\lim \frac{f(P)-f(M)}{M P}= \pm \beta\left(x_{0}, y_{6}\right)
$$

ove vale il $+\mathrm{o}$ il - secondo che $\lim M P$ è equipollente ad $y$ o a $-y$, cioè secondo che $y>y_{0}$ o $y<y_{0}$. 
Posto $\lim M P=y^{\prime}, \lim P_{0} M=x^{\prime}$, sussistono le (18), e dalla (15) segue:

$$
\lim \frac{f(P)-f\left(P_{0}\right)}{P_{0} P}=f_{x^{\prime}}^{\prime}\left(P_{0}\right) \frac{\sin \lambda y^{\prime}}{\sin x^{\prime} y^{\prime}}+f_{y^{\prime}}^{\prime}\left(P_{0}\right) \frac{\sin x^{\prime} \lambda}{\sin x^{\prime} y^{\prime}}
$$

cioè :

$$
\lim \frac{f(P)-f\left(P_{0}\right)}{P_{0} P}=\alpha\left(x_{0}, y_{0}\right) \frac{\sin \lambda y}{\sin x y}+\beta\left(x_{0}, y_{0}\right) \frac{\sin x \lambda}{\sin x y}
$$

Facciamo infine tendere comunque $P$ a $P_{0}$ colla sola condizione $\lim P_{0} P=\lambda$. Designata con $M$ l'intersezione della semiretta $\lambda$ colla parallela all'asse $y$ per $P$ (intersezione certo esistente per $P$ abbastanza prossimo a $P_{0}$, perchè $\lim P_{0} P=\lambda$ ), vale la (15) (dove però $M$ non ha il significato di prima). Se $\lim M P=y^{\prime}$ viene :

$$
\lim \frac{M}{P_{0}} \frac{P}{P}=\frac{\sin \lambda \lambda}{\sin \lambda y^{\prime}}=0, \quad \lim \frac{P_{0} M}{P_{0} P}=\frac{\sin \lambda y^{\prime}}{\sin \lambda y^{\prime}}=1
$$

epperò, essendo limitato il rapporto $\frac{f(P)-f(M)}{M P}$, dalla (15) si trae:

$$
\lim \frac{f(P)-f\left(P_{0}\right)}{P_{0} P}=\lim \frac{f(M)-f\left(P_{0}\right)}{P_{0} M}
$$

Ma pel calcolo del secondo membro si può applicare la (21) (dove $P$ fa le veci di $M$ ); dunque questa relazione vale anche quando $P$ si approssima comunque a $P_{\text {n }}$ sotto la condizione $\lim P_{0} P=\lambda$.

La conclusione è che la (21) vale qualunque sia $\lambda$, anche parallela all'asse $x$ o all'asse $y$, sotto la sola condizione $\lim P_{0} P=\lambda$; epperò tutte le semitangenti ad $F$ in $\bar{P}_{0}$ giaccion sul piano:

$$
z-z_{0}=\alpha\left(x_{0}, y_{0}\right)\left(x-x_{0}\right)+\beta\left(x_{0}, y_{0}\right)\left(y-y_{0}\right),
$$

e quindi (n. 5, Oss.) $f(P)$ ̀े differenziabile in $P_{0}$.

Osservazrone $1^{\text {in }}$. - La condizione indicata non è però necessaria. Per es. la funzione

$$
f(0)=0, \quad f(x)=x^{1+\gamma} \sin \frac{1}{x}(x \neq 0,0<\gamma<1)
$$

concepita come funzione di due variabili, è ovunque differenziabile, perchè $f(x)$ è ovunque derivabile. Ma le sue derivate parziali non sono limitate, perchè il massimo ed il minimo limite di $f^{\prime}(x)$ per $x \rightarrow 0$ sono $+\infty,-\infty$. Pertanto 
in ogni punto dell' asse $y$ i numeri $\alpha(x, y)$. $\beta(x, y)$ relativi a questa funzione non son limitati ('").

Osservazione $2^{\mathrm{a}}$. - Se all'ipotesi che $\alpha(x, y), \beta(x, y)$ sieno limitati nell'interno di $I$ si sostituisce l'ipotesi che esistano in ogni punto interno ad $I$ le derivate $\bar{f}_{x}{ }^{\prime}, \bar{f}_{y}{ }^{\prime}$ e sieno limitate, secondo il teorema di Rademacher citato nella prefazione, si può soltanto affermare che $f(P)$ è differenziabile quasi dappertutto in $I$. Ne è possibile eliminare questa indeterminazione dell' enunciato; perchè è nella natura della cosa, come lo mostra l' esempio della funzione

$$
f(0,0)=0, \quad f(x, y)=\frac{x y}{\sqrt{x^{2}+y^{2}}}
$$

(radicale positivo; $x, y$ non ambedue nulli), la. quale ha derrvate limitate attorno all' origine e non $\dot{e}$ ivi differenziabile $\left({ }^{12}\right)$.

23. La opportunità di sostituire agli ordinari rapporti incrementali parziali i rapporti incrementali generalizzati nello studio delle funzioni di pin variabili, e quindi agli incrementi parziali della funzione gl'incrementi parziali generalizzati, si manifesta evidente anche quando si pon soltanto mente alla continuità superficiale di $f(P)$. Si sa che per la continuità superficiale di $f(l)$ in $P_{0}$ non basta che gl' incrementi $f(x, y)-f\left(x_{0}, y\right), f(x, y)-f\left(x, y_{0}\right)$, $f(x, y)-f\left(x_{0}, y_{0}\right)$ tendano tutti a zero quando $P(x, y)$ tende a $P_{0}$ sulla parallela ad $x$ per $P_{0}$ o sulla parallela ad $y$ per $P_{0}$ o sopra una retta qualunque per $P_{0}$. Invece, come vedremo:

Perchè una funzione $f(x, y)$ sia superficialmente continua in un punto $P\left(x_{0}, y_{0}\right)$ interno ad un dominio piano I dov' essa è definita, bisogna e basta che

$$
\lim \left[f(x, y)-f\left(x_{0}, y\right)\right]=0, \quad \lim \left[f(x, y)-f\left(x, y_{0}\right)\right]=0,
$$

quando $P(x, y)$ tende a $P_{0}\left(x_{0}, y_{0}\right)$ in modo che lim $P_{0} P$ sia una qualunque semivetta $\lambda$, sulla quale $x, y$ rispettivamente non si conservino costanti.

Chiamiamo ancora $M, N$ i punti $\left(x, y_{0}\right),\left(x_{0}, y\right)$. La necessità della condizione è evidente, perchè $f(P)-f(N)=\left[f(P)-f\left(P_{0}\right)\right]+\left[f\left(P_{0}\right)-f(N)\right]$, e analo-

(10) Il ragionamento del $n .21$ prova che quando $f(P)$ è differenziabile in ogni punto interno ad un dominio piano $I$, le possibili rette d'accumulazione in $\bar{P}_{0}$ delle tangenti ad $E^{\prime}$ nei punti d'un intorno di $\bar{P}_{0}$ la cui massima corda tenda a zero, son corde improprie di $F$ in $\bar{P}_{0}$. Percio quando una almeno delle derivate parziali dí $f$ non è limitata attomo a $P_{0}$, vi è in $\bar{P}_{0}$ una corda impropria di $F$ parallela all'asse $z$.

(i) Quest' esempio ed il precedente mi sono stati addotti dal prof. PICone, al quale arevo comunicato i miei risultati. 
gamente per $f(P)-f(M)$. Si vede anzi che i limiti degl'incrementi parziali son zero comunque $P \rightarrow P_{0}$. Ma quel che basta è che i limiti stessi si annullino quando $P \rightarrow P_{0}$ nel modo enunciato.

Ragioniamo per assurdo, supponendo dunque che $f$ non sia superficialmente continua in $P_{n}$. Vi sarà allora ivi un valore d' accumulazione $L$ di $f(P)$, diverso da $f\left(P_{0}\right)$ e in ogni intorno di $P_{0}$ cadranno punti $Q$ tali che $|f(Q)-L| \leq \varepsilon$, ove $2 \varepsilon<\left|L-f\left(P_{0}\right)\right|$.

Da qui, ammessa la forma particolare del postulato di Zermelo, concernente la possibilità di costruire una successione di punti estraendone uno da ciascun insieme di una successione $\left({ }^{12}\right)$, si deduce ovviamente l'esistenza di una successione $P_{\nu}$ convergente a $P_{0}$ e tale che $\left|f\left(P_{y}\right)-L\right| \leq \varepsilon$. Se questa successione ha in $P_{0}$ più d'una semitangente, si potrà (n. 1) estrarre da essa una successione parziale avente in $P_{0}$ una sola semitangente $\lambda$. Continueremo a indicare con $P_{y}$ tale successione parziale. La semitangente $\lambda$ sarà non parallela ad uno almeno, p. es. $y$, dei due assi; e quindi su essa non sarà $x=$ cost..

Designate con $x_{\nu}, y_{v}$ le coordinate di $P_{v}$ e con $N_{v}$ il punto $\left(x_{0}, y_{\nu}\right)$, siccome da un certo $\nu$ in poi l'angolo della semiretta $P_{0} P_{v}$ con $\lambda$ è minore di un angolo comunque prefissato, i punti $P_{\nu}$ saranno distinti dai corrispondenti punti $N_{\nu}$. Si ha ora:

$$
\begin{aligned}
\left|f\left(x_{v}, y_{v}\right)-f\left(x_{0}, y_{\nu}\right)\right| & \geq\left|L-f\left(N_{\nu}\right)\right|-\left|f\left(P_{\nu}\right)-L\right| \geq\left|L-f\left(N_{v}\right)\right|-\varepsilon \\
& \geq\left|L-f\left(P_{0}\right)\right|-\left|f\left(P_{0}\right)-f\left(N_{\nu}\right)\right|-\varepsilon,
\end{aligned}
$$

e siccome, in virtù dell'ipotesi, $f\left(P_{0}\right)-f\left(N_{v}\right)$ tende a zero per $\nu \rightarrow \infty$, così, da un certo $v$ in poi, $\left|f\left(P_{0}\right)-f\left(N_{v}\right)\right|<\varepsilon$ e

$$
\left|f\left(x_{\nu}, y_{v}\right)-f\left(x_{0}, y_{\nu}\right)\right|>\left|L-f\left(P_{0}\right)\right|-2 \varepsilon
$$

che è un nnmero fisso $>0$. Ciò contraddice all'ipotesi di partenza. 亡̀ dunque assurdo ammettere che $f$ non sia superficialmente continua in $P_{0}$.

Osservazione. - Un ragionamento dello stesso tipo, ma più semplice, prova che $f(P)$ è superficialmente continua in $P_{0}$ allora e solo allora che $\lim \left[f(P)-f\left(P_{0}\right)\right]=0$ per $P \rightarrow P_{0}$ in modo che $\lim P_{0} P$ sia una qualunque prefissata semiretta per $P_{0}$, il che si può esprimer dicendo che $f(P)$ è continua nelle diverse direzioni per $P_{0}$. Però questa condizione è molto meno significativa della precedente.

(':) Qui e nell'osservazione del n. 24 son i soli punti della presente Memoria dore si ammette il postalato di ZERMELo (sotto la forma indicata). 


\section{Osservazioni varie.}

24. La nozione di semitangente di un insieme, consente di rendere più espressivo e concreto un teorema relativo ai massimi e minimi di una fun. zione $f(P)$ di due variabili, il quale rimette in valore, nel solo senso in eui essa può rigorosamente sussistere, la vecchia idea di LAGRANGE per la ricerca dei massimi e minimi di $f(P)$, e si applica anche ai massimi e minimi vincolati. Si dimostra infatti facilmente che:

Se $f(P)$ è una funzione reale e continua di un punto $P$ variabile in un insiene piano serrato $I$, per verificare che in un punto d'accumulazione $P_{0}$ di $I$ essa presenta un massimo o un minimo (relativo), basta verificare che $f(P)$ gode di questa proprietà quando $P$ s'avvicina a $P_{0}$ sotto la sola condizione che la semiretta $P_{0} P$ tenda ad una qualunque, ma determinata, semitangente $\lambda$. di $I$ in $P_{0}\left({ }^{13}\right)$.

Ammessa soddisfatta la condizione p. es. pel massimo, si tratta di provare che in un intorno di $P_{0}$ in $I$ è $f(P) \leq f\left(P_{0}\right)$. Se, invero, esistono punti $Q$ di $I$ in ciascun dei quali sia $f(Q)>f\left(P_{0}\right)$, poichè in ogni punto d'accumulazione dei $Q$ la funzione (attesa la sua continuità) assume un valore non minore di $f\left(P_{0}\right)$, i punti $R$ dell'intorno di $P_{0}$ in cui $f(R) \geq f\left(P_{0}\right)$ costituiscono un sottoinsieme serrato $K$ di $I$. L'insieme $K$ avrà in $P_{0}$ almeno una semitan. gente $\lambda$ e si potrà far variare $P$ in una successione $S$ di punti di $K$ con. vergente a $P_{0}$, in modo che $\lim P_{0} l^{\prime}=\lambda$. Si contraddice così all'ipotesi. Pertanto è assurdo ammettere che $f(P)$ non sia massima in $P_{0}$, per $P$ variabile in $T$.

OSSERVAZIONE. - In realtà il ragionamento precedente è esauriente soltanto nel caso in cui $f\left(P_{0}\right)$ sia un massimo o un minimo effettivo (o, come altri dice, proprio), ogni volta che $\lim P_{0} P$ sia una semiretta determinata. Invero, se $f\left(P_{0}\right)$ è sémplicemente un massimo quando $\lim P_{0} P$ è determinato, non si può escludere che nei punti della successione $S$ sia sempre $f(R)=f\left(P_{0}\right)$, epperò non si arriva all' assurdo. Nel caso di un massimo o di un minimo ineffettivo, per concludere sembra necessario di ricorrere al postulato di Zeraelo (sotto la forma particolare applicata nel n. 23). Ammettendo questo

(13) II VIvaNm ("Rend. dei Lincei ", $1898,1^{\circ}$ sem., p. 240 ) ridusse la verifica alla variabilità di $P$ sulle linee cantoriane uscenti da $P_{0}$ (facendo pero uso implicito del postulato di Zermexo). Nella premessa alla Memoria, Sugli estremanti delle funzioni di due variabili ("Memorie della R. Acc. d'Italia », 1930-VIII) io avevo ridotto la verifica alla variabilità di $P$ sulle linee di Jordan uscenti da $P_{0}$. 
postulato basta considerare l'insieme $H$ (non necessariamente serrato) dei punti $Q$ tali che $f(Q)>f\left(P_{0}\right)$ e costruire mediante $H$ una successione di punti $Q$ convergente a $P_{0}$, tale che $f(Q)>f\left(P_{0}\right)$ e $\lim Q P_{0}=\lambda$. Donde l'assurdo.

25. Dato un insieme piano serrato $I$ chiamiamo punto interiore di $I$ ogni punto $P_{\theta}$ tale che le semitangenti ad $I$ in $P_{0}$ sieno a due a due opposte. Quando $I$ è un dominio piano ogni punto interno è interiore. Si prova agevolmente che:

Se $f(P)$ presenta un massimo od un minimo (relativo) in un punto $P_{0}$ interiore all'insieme serrato $I$ dov'essa è definita, ed in $P_{0}$ la funzione è dif. ferenziabile, tutte le derivate direzionali di $f$ son ivi nulle.

Infatti, le derivate direzionali in $P_{1}$ relative a due semitangenti opposte $\lambda,-\lambda$ debbon aver lo stesso valore assoluto e segni contrari, per I'ipotesi della differenziabilità. D'altronde esse risultano ambedue non positive o ambedue non negative, secondochè $f\left(P_{n}\right)$ è un massimo o un minim ; epperò son nulle.

26. Si tenga ora conto del teorema di WeIERstrass per gli estremi di una funzione, nel caso in oui l'insieme piano $I$, dov'essa è data, è un insieme qualunque limitato e serrato (non necessariamente un dominio) $\left({ }^{14}\right)$. Se ne deduce ovviamente che una $f(P)$ continua in ogni punto di un tal insieme, assume in $I$ nn massimo ed un minimo (assoluto). E da ciò segue subito la seguente estensione del teorema di RoLLE $\left(^{15}\right)$.

Se una funzione $f(P)$, finita e continua in tulti $i$ punti di un insieme piano, limitato e serrato, 1 , e differenziabile nei punti interiori, assume valori uguali nei punti non interiori di $I$, esiste qualche punto interiore dove tutte le derivate direzionali son nulle.

Se $z=f(P)$ è una superficie $F$ di Jordar a punti semplici, se ne deduce altresì facilmente che, separata da $F$ una cella con un contorno $l$ a punti semplici (piano o sghembo), esiste almeno un punto $P$ interno alla cella, tale che la proiezione ortogonale di $l$ sul piano tangente in $P$ circonda l'area massima (rispetto alle aree contornate dalle proiezioni ortogonali di $l$ su altri piani dello spazio).

(ii) Ved. le mie Lezioni di Analisi (Bologna, Zanichelli, 1983-XI), p. 171, n. 19.

(15) Estensioni in altro senso si trovano p. es. nelle Lezioni di Analisi infinitesinale di Proone ("Circolo Mat. di Catania", 1928, vol. I), pp. 133, 244. 
27. Le osservazioni precedenti stanno alla base di una trattazione dei massimi e minimi delle funzioni di più variabili, di cui ho appena iniziato lo sviluppo. La considerazione delle derivate direnionali prime evidentemente non basta. Occorre introdurre le derivate direzionali successive. E perciò bisogna anzitutto definire in modo topologico-infinitesimale gl'intorni dei diversi ordini di un punto di accumalazione $O$ di un insieme serrato $I$ di punti di un $S_{1}$. ¿̀ ciò che ho fatto mediante le semiparabole osculatrici (dei varii ordini) ad $I$ nol punto $O$, oppure mediante nna trasformazione birazionale reale, senza eccezioni, dell' intorno di $O$, in guisa da mutare il dato insieme in uno di uno spazio di dimensione tanto alta, che gl'intorni dei varii ordini, da prendersi in considerazione, sieno raggiungibili con spazi lineari uscenti da $O$. Si arriva così alle derivate direzionali dei varii ordini e alla formula di TAYLOR sotto ipotesi molto più ampie delle consuete. Spero di poter tor. nare un'altra volta su quest' argomento.

28. Le nozioni di tangenti e corde improprie di un insieme in un punto di accumulazione permettono di considerare nel gruppo delle trasformazioni topologiche (omeomorfismi) un sottogruppo caratterizzante una geometria intermedia fra la topologia e la geometria differenziale, e che potrebbe chiamarsi topologia del $1^{\circ}$ ordine $\left({ }^{16}\right)$.

Sieno $I, I^{\prime}$ due insiemi perfetti $\left({ }^{17}\right)$ di punti appartenenti a due spazi lineari qualunque; e fra $I, I^{\prime}$ interceda un omeomorfismo $Q$. Si dirà che questo è un omeomorfismo del $1^{\circ}$ ordine quando, considerati due punti $O, O^{\prime}$ di $I, I^{\prime}$, omologhi in $\Omega$, l'omeomorfismo fa corrispondere biunivocamente ad ogni semitangente o semicorda impropria di $I$ in $O$ una semitangente o rispettivamente semicorda impropria di $I^{\prime}$ in $O^{\prime}$, ad ogni tangente e ad ogni corda impropria, una tangente od una corda impropria. Con ciò si vuol dire che, mentre $P$ tende in $I$ a $O$ in guisa che $\lim O P=\lambda, P^{\prime}$ tende in $I^{\prime}$ ad $O^{\prime}$ in guisa che la semiretta $O^{\prime} P^{\prime}$ tende essa pure verso un limite determinato $\lambda^{\prime}$; e che mentre $P, Q$ tendono in $I$ a $O$ in modo che $\lim P Q=\mu, P^{\prime}, Q^{\prime}$ tendono in $I^{\prime}$ ad $O^{\prime}$ in guisa che la semiretta $P^{\prime} Q^{\prime}$ tende anch' essa verso un limite determinato $\mu^{\prime}$. Inoltre a semitangenti o semicorde opposte corrispondono semitangenti e semicorde opposte.

(16) $\mathrm{E}$ in sostanza la stessa geometria avente come gruppo fondamentale il gruppo topologico ristretto. di cui al n. 67 del libro di Bovligaxd.

(17) Si puo anche supporre soltanto che $I, I^{\prime}$ siano serrati, bastando allora riferire la definizione suecessiva a coppie di punti omologhi che siano di accumulazione pei due insiemi. 
Le operazioni di proiezione entro uno spazio lineare $S_{n}$ dànno generalmente lnogo ad omeomorfismi del to ordine. Sia $O$ un punto di accumulazione di un insieme serrato $I$ di $S_{n}$ e sia $C$ un centro di proiezione. Allora condiaione necessaria e sufficiente perchè fra l'intomo di $O$ in $I$ e l'intono del punto $O^{\prime}$ nell' insieme $I$ proiezione di $I$ da $O$ sopra un iperpiano, vi sia un omeomorfismo del $t^{\circ}$ ordine, $\grave{e}$ che le corde improprie di $I$ in $O$ stieno tutte (almeno) in un iperpiano non passante per $C$.

L'asserzione è di verifica immediata.

Si può così parlare di dimensione di un insieme serrato $I$ in un suo punto d' accumulazione $O$ come della dimensione dello spazio lineare minimo in oui si può proiettare l'intorno di $O$ in $I$, in guisa che l'intorno proiezione sia omeomorfo del $1^{\circ}$ ordine al dato. È chiaro che la dimensione $k$ di $I$ in $O$ uguaglia la dimensione del minimo spazio lineare congiungente le corde improprie di $I$ in $O$ (le quali, è superfluo dirlo, non costituiscono però sempre uno spazio lineare!) ( $\left.{ }^{18}\right)$. E altresi evidente che la dimensione di accumulazione di una $M_{k}$ topologica in un suo punto uguaglia $k$ e supera $k$ secondo che il punto di $M_{k}$ è semplice o no.

Non sembra facile rispondere alla questione se la dimensione $k$ di un insieme serrato $I$ in un suo punto d'accumulazione $O$ sia o meno un invariante di fronte agli omeomorfismi del $1^{\circ}$ ordine (la cosa è ovvia per $k=1$ ). Possiamo invece agevolmente dimostrare che:

Il concetto di punto semplice di una varietà topologica è invariante di fronte agli omeomorfismi del $1^{\circ}$ ordine.

Esponiamo la dimostrazione, per brevità di linguaggio, nel caso di una superficie $F$. Premettiamo che, se due linee di JoRdan $l, m$ di $S_{n}$ aventi in $O$ un punto semplice, non si toccano ivi, l'insieme $l+m$ ha come corde improprie in $O$ tutte le rette per $O$, appartenenti al piano $\omega$ delle due tangenti. Indichiamo invero con $H_{\S}$ l'insieme delle rette che conginngono coppie di punti distinti $P, Q$ di $l+m$, situati nell'intorno di raggio $\varepsilon$ di $O$. Se una retta $a$ per $O$ è di accumulazione per $H_{\varepsilon}$ qualunque sia $\varepsilon$, ed esistono rette $P Q$ formanti con $\alpha$ un angolo comunque piccolo ed i cui punti $P, Q$ variano o su $l$ o su $m$, la $a$ coincide colla tangente in $O$ ad $l o$ ad $m$. Se invece esistono rette $l^{\prime} Q$ formanti con $a$ un angolo comunque piccolo ed i cui punti $P, Q$

(18) Il Guareschi nel lavoro citato introduce la locuzione di "dimensione di un' accumu. lazione $O$ "come numero delle tangenti linearmente indipendenti in $O$. Ma un insieme $I$ di $S_{n}$, the abbia in $O$ un'accumulazione di dimensione $n-1$ in questo senso, può benissimo non esser biunivocamente proiettabile (nell'intorno di 0 ) sopra un iperpiano. 
distinti fra loro e da $O$ stieno uno su $l$ e l'altro su $m$, il piano $O P Q$ lindividuato per $\varepsilon$ abbastanza piccolo!) ha per limite il piano $\omega$ e quindi $a$ giace in $\omega$.

Viceversa, ogni retta $a$ del fascio $O$ in $\omega$ è corda impropria, perchè, se è distinta dalle tangenti in $O$ ad $l, m$ (che si sa già esser corde improprie), condotto per a un iperpiano $S_{n-1}$, che nou contenga $\omega$, ogni iperpiano parallelo a quello e ad esso abbastanza vicino, sega $l$, $m$, attorno ad $O$, ciascuna in un punto e la congiungente di questi due punti ha per limite $\alpha$.

Ciò premesso, sia $F^{\prime}$ la trasformata di $F$ mediante un omeomorfismo $\mathbf{Q}$ del $1^{\circ}$ ordine fra gl'intorni dei punti omologhi $O, O^{\prime}$ di $F, F^{\prime}$. Su $F^{\prime}$ si posson intanto tracciare infinite linee di JORDAN, aventi in $O^{\prime}$ un punto semplice; le trasformate mediante $\Omega$ delle linee analoghe uscenti da $O$ su $F$. Anzi esistono in $F^{\prime}$ linee di JoRDAN passanti semplicemente per $O^{\prime}$ con un'assegnata tangente. Per ogni coppia di linee di Jondas uscenti su $F^{\prime}$ da $O^{\prime}$, con tangenti distinte, si hanno $\infty^{1}$ corde improprie di $F^{\prime}$ in $O^{\prime}$ situate sul piano delle due tangenti. Pertanto tatte le corde improprie di $F^{\prime}$ giacciono in questo piano, che non muta al variar delle due linee, se no non ci potrebbe esser corrispondenza biunivoca fra le corde improprie di $F$ e le corde improprie di $F^{\prime}$ in $O, O^{\prime}$. Dunque $O^{\prime}$ è semplice per $F^{\prime \prime}$.

29. La corrispondenza indolta da un omeomorfismo del $1^{\circ}$ ordine fra le tangenti in due punti semplici omologhi di due varietc̀ topologiche di dimensione $k$, è proieltiva.

Questa è conseguenza ovvia del teorema precedente, per $k>2$. Invero, se $O . O^{\prime}$ son i due punti corrispondenti delle varietà $M_{k}, M_{k}^{\prime}$, la corrispondenza indotta fra le due stelle di tangenti omologhe in $O, O^{\prime}$ è proiettiva, perchè è binnivoca e ad un fascio di rette fa corrispondere un fascio di rette.

Suppongasi $k=2$, e si osservi anzitutto che quando la superficie $F$, avente in $O$ un punto semplice, appartiene ad uno spazio $S_{r}(r \geqq 3)$, si può sempre proiettarla da un $S_{r-3}$, che non incontri il piano tangente in $O$ (epperò nessuna corda impropria di $F$ ), sopra un piano. La corrispondenza $\Omega$ fra l'intorno di $O$ in $F$ e l'intorno piano del punto omologo $O^{\prime}$ è un omeomorfismo del $1^{\circ}$ ordine (n. 28) e la corrispondenza indotta fra le tangenti in $O, O^{\prime}$ ai due insiemi è proiettiva, perchè generata da una proiezione.

Sicchè tutto si riduce a provare che un omeomorfismo del $1^{\circ}$ ordine $\mathbf{Q}$ fra due intorni piani $\pi, \pi^{\prime}$, di centri $O, O^{\prime}$, dà luogo ad un'omografia fra le direzioni omologhe. Infatti le coordinate $x^{\prime}, y^{\prime}$ di un punto mobile in $\pi^{\prime}$ risultano funzioni continue delle coordinate $x, y$ del punto corrispondente in $\pi$, le quali, a cagion delle proprietà di $Q$, ammettono in $O^{\prime}$ iperderivate in tutte 
le direzioni. Perciò le $x^{\prime}, y^{\prime}$ sono differenziabili (anzi iperdifferenziabili). In conseguenza il coefficiente angolare di una direzione uscente da $O^{\prime}$ è funzione lineare del coefficiente angolare dell'omologa direzione per 0 .

Un elegante corollario delle precedenti considerazioni è questo:

Condizione necessaria e sufficiente perchè una $f(P)$ di un punto $P$ mobile in un doninio piano $I$, sia iperdifferenziabile in un punto $P_{0}$ interno ad $I$, ̀̀ che fra $i$ punti di un intorno di $P_{0}$ e $i$ corrispondenti valori di $f(P)$ vi sia un omeomorfismo del $1^{\circ}$ ordine.

OSSERVAZIONE. - Si posson altresi considerare omeomorfismi di ordine superiore definiti in relazione agl'intorni topologico-infinitesimali dei varii ordini di un punto di accumulazione di un insieme. 